\title{
AN EXTENSION OF THE SEWING LEMMA TO HYPER-CUBES AND HYPERBOLIC EQUATIONS DRIVEN BY MULTI PARAMETER YOUNG FIELDS
}

\author{
FABIAN A. HARANG \\ UNIVERSITY OF OSLO
}

\begin{abstract}
This article extends the celebrated sewing lemma to multi-parameter fields on hyper cubes. We use this to construct Young integrals for multi-parameter Hölder fields on general domains $[0,1]^{k}$ with $k \geq 1$. Moreover, we show existence, uniqueness and stability of some particular types of hyperbolic PDE's driven by space-time Hölder noise in a Young regime.
\end{abstract}

\section{Contents}

1. Introduction

2. Notation and framework

3. Sewing Lemma and Young integration for fields

4. Differential equations driven by Young Fields

References

\section{INTRODUCTION}

In recent years, analysis of stochastic processes based on their path-wise properties rather than their probabilistic properties has received much attention. Especially after T. Lyons published the celebrated article on rough paths $([8])$ in the late 1990's, this field of research exploded. He proved that if one can show (by probabilistic methods) that for a $\alpha$-Hölder continuous stochastic process $X$ with $\alpha \in\left(\frac{1}{3}, \frac{1}{2}\right]$ there exists a Lèvy area (iterated integral) corresponding to $X$ satisfying some algebraic and analytical conditions, one can give path-wise solutions to SDE's of the form

$$
d Y_{t}=f\left(Y_{t}\right) d X_{t},
$$

where $f$ is sufficiently smooth. This is an important extension of similar results known when the regularity parameter $\alpha$ of $X$ is above $\frac{1}{2}$, where Young integration theory can be used to prove that a unique solution to Equation (1.1) exists. The main advantage of the path-wise approach to stochastic calculus is that it does not rely upon martingale properties of the driving signal, but rather depend on its Hölder continuity.

The current article was motivated by the construction of a path-wise rough integration method for stochastic fields in the framework of the theory of rough paths. However, as we shall see, the study of multi-parameter processes requires much more detail than the 1D theory of SDE's, and we therefore have chosen to divide this project into two parts. This is the first part, and here we

Key words and phrases. Young integrals, Sewing lemma, random fields, multi-parameter integration, hyperbolic SPDE's, stochastic partial differential equations, path-wise integration. MSC classification : 35L05, 60H15, 60H05, 60H20.

Acknowledgment: I am very are grateful for all the assistance, comments and advice I have received from Professor Frank N. Proske during the work on this project. Also, I would like to thank Professor Fred E. Benth and Professor Samy Tindel, whose comments greatly improved this manuscript. 
will focus on extending the sewing lemma to general Hölder fields on domains of the form $[0,1]^{k}$ for $k \geq 1$. We will apply this extension to show how we can construct Young integrals for multiparameter Hölder fields. At last, we prove existence and uniqueness of some hyperbolic SPDE's with quite general boundary conditions, driven by Hölder noise in a Young regime. The second article is an ongoing work to provide a framework similar to that of rough paths for fields based on the sewing lemma that we provide in this article.

Consider two stochastic fields $X, Y:[0,1]^{k} \rightarrow \mathbb{R}$, with some Hölder continuity assumption in each of its variables, in the sense that for each $i \in\{1, \ldots, k\}$ we have estimates of the form

$$
\sup _{\left(x_{1}, \ldots, x_{k}\right) \in[0,1]^{k}, y_{i} \in[0,1]}\left|X\left(x_{1}, \ldots, y_{i}, \ldots, x_{k}\right)-X\left(x_{1}, \ldots, x_{i}, \ldots, x_{k}\right)\right| \lesssim\left|y_{i}-x_{i}\right|^{\alpha_{i}},
$$

for some $\alpha \in(0,1)^{k}$ and similarly for $Y$ with respect to some $\beta \in(0,1)^{k}$. We are interested in a path-wise construction of integrals of the form

$$
\int_{x_{1}}^{y_{1}} \cdots \int_{x_{k}}^{y_{k}} Y\left(z_{1}, \ldots, z_{k}\right) X\left(d z_{1}, \ldots, d z_{k}\right)
$$

where the differential (at least when $X$ is smooth) can be interpreted as

$$
X\left(d z_{1}, \ldots, d z_{k}\right)=\frac{\partial^{k}}{\partial x_{1}, \ldots, \partial x_{k}} X\left(z_{1}, \ldots, z_{k}\right) d z_{1}, \ldots, d z_{k} .
$$

Integrals of this form have been studied from a random field point of view see e.g. [22], but also other meanings have been given to such integrals, e.g. see [11. In recent years, integrals on the form of Equation (1.3) are often referred to as integration with respect to Ambit fields, where $X$ usually is chosen to be some multi-parameter Volterra field of the form

$$
X\left(y_{1}, \ldots, y_{k}\right)=\int_{A\left(y_{1}, . ., y_{k}\right)} G\left(y_{1}-z_{1}, \ldots, y_{k}-z_{k}\right) L\left(d z_{1}, \ldots, d z_{k}\right),
$$

where $L$ is a Lèvy basis, $G$ is a possibly singular Volterra kernel, and $A\left(y_{1}, . ., y_{k}\right)$ is some subset of $[0,1]^{k}$ depending on $\left(y_{1}, \ldots, y_{k}\right)$, see for example [21]. If $L$ is chosen to be a Brownian sheet, and the resulting field $X$ satisfy Equation (1.2) for some $\alpha \in(0,1)^{k}$, Young type integration with respect to $X$ has been developed by Tindel and Quer-Sardanyons in [17, and also by N. Towghi in 18 for two dimensions. That is, both [17] and [18] shows that for $k=2$, if $X$ and $Y$ satisfy Equation (1.2) for Hölder coefficients $\left(\alpha_{1}, \alpha_{2}\right)$ and $\left(\beta_{1}, \beta_{2}\right)$ respectively with $\alpha_{1}+\beta_{1}>1$ and $\alpha_{2}+\beta_{2}>1$ then integrals of the form

$$
\begin{gathered}
\iint_{[s, t] \times[x, y]} Y\left(z_{1}, z_{2}\right) X\left(d z_{1}, d z_{2}\right) \\
:=\lim _{|\mathcal{P}[s, t] \times \tilde{\mathcal{P}}[x, y]| \rightarrow 0} \sum_{[u, v] \in \mathcal{P}} \sum_{[\tilde{u}, \tilde{v}] \in \tilde{\mathcal{P}}} Y(u, \tilde{u}) \int_{u}^{v} \int_{\tilde{u}}^{\tilde{v}} X(d \mathbf{z}),
\end{gathered}
$$

exists, and behaves like a two parameter extension of the Young integral. The former authors apply this 2-d Young integral to study the stochastic wave equation driven by a fractional Brownian sheet, with regularity above $\frac{1}{2}$ in each variable. Our aim is to extend this integration from $[0,1]^{2}$ to $[0,1]^{k}$ by extending the Sewing lemma familiar from rough paths theory.

In the seminal paper "Rough Sheets" by M. Gubinelli and K. Chouk ([3]) from 2014, the authors studied such integration with the motivation to analyze the stochastic wave equation in a rough path framework. Their methodology is based on the insightful "algebraic integration" framework developed by Gubinelli in previous works (see e.g. [4, 9] for an introduction). However, as the authors of [3] point out;

- "Unfortunately the construction of a rough integral in a multi-parameter setting gives rise to a very complicated algebraic structure which is not well understood at the moment."

The article is therefore restricted only to the construction of the integral, and do not deal with the 
stochastic wave equations, or other forms of differential equations. Their construction of the integral in $2 \mathrm{D}$ is also based on an extension of the sewing lemma, but proven using the framework of the algebraic integration theory. Our approach is based on a direct extension of Lemma 4.2 in [13].

The crucial part of this procedure is the construction of a $\delta$ operator acting on functions $\Xi$ : $[0,1]^{k} \times[0,1]^{k} \rightarrow \mathbb{R}$, which is used to prove the existence of an abstract integration map $\mathcal{I}$ constructed as a limit of a Riemann type sum with respect to the functions $\Xi$ of $\alpha \in(0,1)^{k}$ joint-Hölder continuity. That is, for $\mathbf{x}, \mathbf{y} \in[0,1]^{k}$ let $\mathcal{P}\left[x_{i}, y_{i}\right]$ be a partition of $\left[x_{i}, y_{i}\right]$ for $i \in\{1, . ., k\}$ and set

$$
\begin{gathered}
\mathcal{I}(\Xi)(\mathbf{x}, \mathbf{y})=\lim _{|\mathcal{P}[\mathbf{x}, \mathbf{y}]| \rightarrow 0} \sum_{[\mathbf{u}, \mathbf{v}] \in \mathcal{P}} \Xi(\mathbf{u}, \mathbf{v}) \\
=\lim _{\left|\mathcal{P}\left[x_{1}, y_{1}\right]\right| \vee, \ldots, \vee\left|\mathcal{P}\left[x_{k}, y_{k}\right]\right| \rightarrow 0} \sum_{\left[u_{1}, v_{1}\right] \in \mathcal{P}\left[x_{1}, y_{1}\right]}, \ldots, \sum_{\left[u_{k}, v_{k}\right] \in \mathcal{P}\left[x_{k}, y_{k}\right]} \Xi\left(u_{1}, \ldots, u_{k}, v_{1}, \ldots, v_{k}\right),
\end{gathered}
$$

where $\Xi$ is assumed to satisfy some regularity conditions and algebraic relations with respect to the operator $\delta$. The main result of this sewing Lemma is that we obtain an inequality of the form

$$
\left|\mathcal{I}(\Xi)_{\mathbf{x}, \mathbf{y}}-\Xi(\mathbf{x}, \mathbf{y})\right| \lesssim\|\delta \Xi\|_{\beta ;[0,1]^{k}} q_{\alpha}(\mathbf{x}, \mathbf{y}) p_{\beta-\alpha}(\mathbf{x}, \mathbf{y}) ; \mathbf{x}, \mathbf{y} \in[0,1]^{k}
$$

where $\beta \in(1, \infty)^{k}$ and $q_{\alpha}(\mathbf{x}, \mathbf{y}):=\prod_{i=1}^{k}\left|x_{i}-y_{i}\right|^{\alpha_{i}}$, and $p_{\beta-\alpha}(\mathbf{x}, \mathbf{y}):=\sum_{i=1}^{k}\left|x_{i}-y_{i}\right|^{\beta_{i}-\alpha_{i}}$, and the Hölder type norm will be defined properly in the next section. This type of inequality tells us that if the action of $\delta$ on $\Xi$ is sufficiently regular, then $\Xi$ is a sufficiently good local approximation of the integral $\mathcal{I}(\Xi)$. As a consequence, if we introduce more terms to regularize $\Xi$ it will not affect the limit of the Riemann type sum. by

If $X$ satisfies inequality (1.2) for $1 \leq i \leq k$ and $\alpha \in\left(\frac{1}{3}, \frac{1}{2}\right]^{k}$, then we define the integral in (1.3)

$$
\int_{x_{1}}^{y_{1}} \cdots \int_{x_{k}}^{y_{k}} f\left(X\left(z_{1}, \ldots, z_{k}\right)\right) X\left(d z_{1}, \ldots, d z_{k}\right):=\mathcal{I}(\Xi)_{\mathbf{x}, \mathbf{y}},
$$

where $\Xi(\mathbf{x}, \mathbf{y})=f(X(\mathbf{x})) \square_{\mathbf{x}, \mathbf{y}} X$ where $\square_{\mathbf{x}, \mathbf{y}} X=\int_{x_{1}}^{y_{1}} \cdots \int_{x_{k}}^{y_{k}} X\left(d z_{1}, \ldots, d z_{k}\right)$ denotes the rectangular (or generalized) increment of $X$, which will be described in detail in the next section.

With the construction of a Young integral, we continue to show existence on uniqueness of differential equations driven by Hölder fields on hyper cubes, that is; for $X:[0,1]^{k} \rightarrow \mathbb{R}$, we show existence and uniqueness of the equation

$$
Y(\mathbf{x})=\xi(\mathbf{x})+\int_{0}^{x_{1}} \cdots \int_{0}^{x_{k}} f(Y(\mathbf{z})) X(d \mathbf{z}), \quad \mathbf{x} \in[0,1]^{k},
$$

where $\mathbf{x} \mapsto \xi(\mathbf{x})$ is assumed to be some Hölder contiguous field, and captures the behavior of the above equation on the boundary. Such equations can be viewed as a stochastic extension of problems connected to hyperbolic PDE's that are given by the following mixed derivative equation

$$
\partial_{x, y} u=b\left(x, y, u, \partial_{x} u, \partial_{y} u\right),(x, y) \in[0,1]^{2}
$$

subject to the boundary conditions

$$
\begin{gathered}
u(x, 0)=f(x) \\
u(0, y)=g(y) \\
f(0)=g(0),
\end{gathered}
$$

for some sufficiently smooth functions $b, f$, and $g$. A particularly simple example would be to choose $b\left(x, y, u, \partial_{x} u, \partial_{y} u\right)=b(u)$, and by integrating both sides, we obtain

$$
u(x, y)=-u(0,0)+f(x)+g(y)+\int_{0}^{x} \int_{0}^{y} b\left(u\left(z_{1}, z_{2}\right)\right) d z_{1} d z_{2} .
$$

If $b$ is Lipschitz, classical analysis arguments give us existence and uniqueness of this equation. 
Furthermore, we can introduce randomness in the above equation by letting $b$ be multiplied by a space time white noise $\partial_{x, y} W$. This results in the non-linear (multiplicative) hyperbolic SPDE

$$
\partial_{x, y} u=b(u) \partial_{x, y} W, \quad(x, y) \in[0,1]^{2},
$$

where we assume that it is subject to the same boundary conditions as earlier. This equation can be written in integral form

$$
u(x, y)=-u(0,0)+f(x)+g(y)+\int_{0}^{x} \int_{0}^{y} b\left(u\left(z_{1}, z_{2}\right)\right) W\left(d z_{1}, d z_{2}\right) .
$$

Such equations have received a lot of attention in probability theory for the last 30-40 years, and is tightly connected to the wave equation, as described in [17]. For example Walsh studied this type of integral equation in a classical probability setting, using the probabilistic properties of the Brownian sheet $W$ (see 22]). With the framework presented in the current article, we are able to study such problems without using probability theory, by considering equations on the form

$$
\frac{\partial^{k}}{\partial x_{1}, \ldots, \partial x_{k}} u=b(u) \frac{\partial^{k}}{\partial x_{1}, \ldots, \partial x_{k}} X, \quad(x, y) \in[0,1]^{k},
$$

where $X \in \mathcal{C}^{\alpha}\left([0,1]^{k} ; \mathbb{R}\right)$ for $\alpha \in\left(\frac{1}{2}, 1\right)^{k}$ and $\frac{\partial^{k}}{\partial x_{1}, \ldots, \partial x_{k}} X$ is understood as a distributional derivative, and we study the mild form of this equation given by

$$
u(\mathbf{x})=\xi(\mathbf{x})+\int_{\mathbf{0}}^{\mathbf{x}} b(u(\mathbf{z})) X(d \mathbf{z}),
$$

where the boundary information is collected in $\xi$. We want to note that this type of equations can be viewed as an extension of the equations studied on $[0,1]^{2}$ in [17] but with more general boundary.

An interesting note when we study Young integration with respect to fields is that both [17, 18, study Equation (1.3) in a Young type of setting, and give a definition of this integral in $[0,1]^{2}$. This 2D young integral has been used by P. Friz et. al to give a canonical construction of iterated integrals for Gaussian processes with sufficiently nice co-variance structure (see [15, 13, 12, for a detailed exposition in this direction). For example, the fact that one can only construct (canonically) the iterated integral of an $\mathrm{fBm} B^{H}$ with Hurst parameter $H>\frac{1}{4}$ is proved in this manner. This bound is based on the need for a type of complementary Young regularity of the co-variance function of the $\mathrm{fBm}$, in the sense that if $Q:[0,1]^{4} \rightarrow \mathbb{R}$ is the co-variance function of a $\mathrm{fBm}$, then $Q$ is of $2 H$ regularity, and we need that $2 H+2 H>1$ for the construction of $\int Q d Q$ in a Young regime. If we have a proper construction of $\int Q d Q$ we can bound the variance of $\int B^{H} d B^{h}$ (constructed in a Riemann type way) by the variation norm of $\int Q d Q$. With the construction of the sewing Lemma in the current article, we believe that a similar study of the regularity of co-variance functions of Gaussian fields will make us able to construct iterated integrals (and similar polynomial concepts as we will discuss later) of said fields. In particular if $X:[0,1]^{k} \rightarrow \mathbb{R}^{d}$ is a Gaussian field, then the co-variance will be $Q:[0,1]^{2 k} \rightarrow \mathbb{R}^{d \times d}$, and if this function has sufficient regularity, we believe one can construct an integral of the form $\int Q d Q$ in a Young type way over $[0,1]^{2 k}$. However, we have not yet had the time to explore this yet, but hope to do this in the future.

This article is structured as follows:

- Section 1 and 2 give a general introduction to the problem at hand and the framework we use, as well as notation. We propose a generalization of the $\delta$ operator known from rough path theory, and show how we can decompose this operator into more simple operators to use in analysis later.

- Section 3 extends the sewing Lemma and describes how we can use this to construct a Young integral for general fields of the form $X:[0,1]^{k} \rightarrow \mathbb{R}$ for $k \geq 1$ of some Hölder continuity. 
- Section 4 is devoted to the existence and uniqueness of differential equations driven by Young fields with Hölder coefficients $\alpha$ in $\left(\frac{1}{2}, 1\right)^{k}$. Stability results are provided with respect to the solution.

\section{Notation AND FRAMEWORK}

Throughout this article, we will work on subsets of $[0,1]^{k}$ for some $k \geq 1$. We will mostly work on subsets of the form $[\mathbf{x}, \mathbf{y}]=\prod_{i=1}^{k}\left[x_{i}, y_{i}\right]$, where $\mathbf{x}=\left(x_{1}, . ., x_{k}\right) \in[0,1]^{k}$ and similarly for $\mathbf{y}$. We will frequently write for two elements $\mathbf{x}, \mathbf{y} \in[0,1]^{k}$ that $\mathbf{x}<\mathbf{y}$, where we mean $x_{i}<y_{i} \forall i=1, . ., k$. In the same way, we may use the notation $\mathbf{x} \leq \mathbf{y}$ for the criterion $x_{i} \leq y_{i} \forall i=1, . ., k$. When working with integration over hyper cube domains we will call a domain degenerate if one or more of the intervals which constitutes the hyper cube has zero length. The reason is that if we have a hyper cube $[\mathbf{x}, \mathbf{y}]=\prod_{i=1}^{k}\left[x_{i}, y_{i}\right]$ where for some $1 \leq i \leq k, x_{i}=y_{i}$ we have formally for some integrable function $g$ that

$$
\int_{[\mathbf{x}, \mathbf{y}]} d g=\int_{x_{1}}^{y_{1}} \cdots \int_{x_{i}}^{x_{i}} \cdots \int_{x_{k}}^{y_{k}} d g=0 .
$$

Thus, we often specify that the interval of interest $[\mathbf{x}, \mathbf{y}]$ must satisfy $\mathbf{x}<\mathbf{y}$. Most computations in this article are based on a generalized increment on hyper cubes. We therefore give the following definition of an operator generating increments of functions in $[0,1]^{k}$.

Definition 1. (generalized increments) For $\mathbf{x}=\left(x_{1}, . ., x_{k}\right) \in[0,1]^{k}$ and $\mathbf{y}=\left(y_{1}, . ., y_{k}\right) \in[0,1]^{k}$ we define the operator $V_{i, \mathbf{x}}$ for each $i \in\{1, \ldots, k\}$ by

$$
V_{i, \mathbf{x}} \mathbf{y}=\left(y_{1}, . ., y_{i-1}, x_{i}, y_{i+1}, . ., y_{k}\right) \text {. }
$$

For a function $f:[0,1]^{k} \rightarrow \mathbb{R}$ we define the generalized increments of $f$ from $\mathbf{x}$ to $\mathbf{y}$ (i.e over $\left.[\mathbf{x}, \mathbf{y}] \subset[0,1]^{k}\right)$ by

$$
\square_{\mathbf{x}}^{k} f(\mathbf{y})=\prod_{i=1}^{k}\left(I-V_{i, \mathbf{x}}\right) f(\mathbf{y})
$$

where $I$ denotes the identity operator, and $V_{i, \mathbf{x}} f(\mathbf{y})=f\left(V_{i, \mathbf{x}} \mathbf{y}\right)$. Moreover, if $f$ is a smooth field, we have

$$
\int_{\mathbf{x}}^{\mathbf{y}} f(d \mathbf{z})=\int_{\mathbf{x}}^{\mathbf{y}} \frac{\partial^{k}}{\partial z_{1}, \ldots, \partial z_{k}} f(\mathbf{z}) d \mathbf{z}=\square_{\mathbf{x}}^{k} f(\mathbf{y})
$$

Remark 2. It will be convenient for later to use the notation for product of generalized increments

$$
\begin{aligned}
\square_{\mathbf{x}}^{n} f(\mathbf{y}) \square_{\tilde{\mathbf{x}}}^{n} f(\tilde{\mathbf{y}})= & \prod_{k=1}^{n}\left(I-V_{k, \mathbf{x}}\right) \prod_{k=1}^{n}\left(I-V_{k, \tilde{\mathbf{x}}}\right) f(\mathbf{y}) f(\tilde{\mathbf{y}}) \\
& =\square_{(\mathbf{x}, \tilde{\mathbf{x}})}^{2 n} f(\mathbf{y}) f(\tilde{\mathbf{y}}),
\end{aligned}
$$

i.e. we use $\square_{(\mathbf{x}, \tilde{\mathbf{x}})}^{2 n}=\prod_{k=1}^{2 n}\left(I-V_{k,(\mathbf{y}, \tilde{\mathbf{y}})}\right)$ acting on functions on $\left(\mathbb{R}^{n}\right) \times\left(\mathbb{R}^{n}\right)$ in the above way.

Also notice the inductive property of the generalized increment, that is

$$
\begin{aligned}
\square_{\mathbf{x}}^{n} f(\mathbf{y})= & \prod_{k=1}^{n-1}\left(I-V_{k, \mathbf{x}}\right) f(\mathbf{y})-V_{n, \mathbf{x}} \prod_{k=1}^{n-1}\left(I-V_{k, \mathbf{x}}\right) f(\mathbf{y}) \\
& =\square_{\mathbf{x}^{\prime}}^{n-1} f\left(\mathbf{y}^{\prime}, y_{n}\right)-\square_{\mathbf{x}^{\prime}}^{n-1} f\left(\mathbf{y}^{\prime}, x_{n}\right),
\end{aligned}
$$

which will become very useful in the later sections.

For a more compact notation, write $\square_{\mathbf{x}, \mathbf{y}}^{k} f=\square_{\mathbf{x}}^{k} f(\mathbf{y})$ as the $k$ increment of $f$ from $\mathbf{x}$ to $\mathbf{y}$. 
Example 3. Consider a function $f(\mathbf{x})=\prod_{i=1}^{k} f_{i}\left(x_{i}\right)$, where $f_{i}:[0,1] \rightarrow \mathbb{R}$ is differentiable. Then

$$
\square_{\mathbf{x}, \mathbf{y}}^{k} f=\prod_{i=1}^{k}\left(f_{i}\left(y_{i}\right)-f_{i}\left(x_{i}\right)\right) .
$$

Also notice that by the mean value Theorem we have

$$
\square_{\mathbf{x}, \mathbf{y}}^{k} f=\frac{\partial^{k}}{\partial x_{1}, \ldots, \partial x_{k}} f(\mathbf{c}) \prod_{i=1}^{k}\left(y_{i}-x_{i}\right)
$$

for some $\mathbf{c} \in[\mathbf{x}, \mathbf{y}]$, the last relation holds of course for general $f:[0,1]^{k} \rightarrow \mathbb{R}$ which is at least one time differentiable in each variable on $[0,1]^{k}$.

There are two ways to consider Hölder continuous functions in several variables. One could either consider the space of functions which satisfy a Hölder continuity condition on each sub-interval of a hyper cube, or we could define it as the space of functions whose generalized increment $\square$ satisfies some multiplicative Hölder type of regularity. In the current article we will use both concepts, and will therefore give a definition of a space which captures both.

Definition 4. Let $\alpha \in(0,1)^{k}$ be a multi-index. The space of Hölder continuous fields $\mathcal{C}^{\alpha}\left([0,1]^{k} ; \mathbb{R}\right)$ is defined as all $Y:[0,1]^{k} \rightarrow \mathbb{R}$ which satisfy

$$
\|Y\|_{\alpha,[0,1]^{k}}:=\|Y\|_{\alpha, \square,[0,1]^{k}}+\sum_{i=1}^{k} \sup _{\mathbf{x} \in[0,1]^{k}}\left\|Y_{\mathbf{x}, i}\right\|_{\alpha_{i} ;[0,1]}<\infty,
$$

where $Y_{\mathbf{x}, i}=Y\left(x_{1}, . ., x_{i-1}, \cdot, x_{i+1}, . ., x_{k}\right)$ and

$$
\begin{gathered}
\left\|Y_{\mathbf{x}, i}\right\|_{\alpha_{i} ;[0,1]}=\sup _{u \neq v \in[0,1]} \frac{\left|Y_{\mathbf{x}, i}(v)-Y_{\mathbf{x}, i}(u)\right|}{|v-u|^{\alpha_{i}}} \\
\|Y\|_{\alpha, \square,[0,1]^{k}}=\sup _{\mathbf{x}, \mathbf{y} \in[0,1]^{k} \backslash D} \frac{\left|\square_{\mathbf{x}, \mathbf{y}}^{k} Y\right|}{q_{\alpha}(\mathbf{x}, \mathbf{y})},
\end{gathered}
$$

where $q_{\alpha}(\mathbf{x}, \mathbf{y})=\prod_{i=1}^{k}\left|y_{i}-x_{i}\right|^{\alpha_{i}}$, and

$$
D=\left\{\mathbf{x} \in[0,1]^{k} \mid x_{i} \neq x_{j} \text { for } i \neq j \in\{1, . ., k\}\right\} .
$$

Also, let us denote by

$$
\|Y\|_{\alpha,+,[0,1]^{k}}=\sum_{i=1}^{k} \sup _{\mathbf{x} \in[0,1]^{k}}\left\|Y_{\mathbf{x}, i}\right\|_{\alpha_{i} ;[0,1]},
$$

i.e. the Hölder semi norm, but without the multiplicative regularity part.

From the above we can see that

$$
\|Y\|_{\infty}:=\sup _{\mathbf{x} \in[0,1]^{k}}|Y(\mathbf{x})| \leq|Y(\mathbf{0})|+\|Y\|_{\alpha,+,[0,1]^{k}},
$$

which will become useful later.

Proposition 5. For $Y \in \mathcal{C}^{\alpha}\left([0,1]^{k} ; \mathbb{R}\right)$ the mapping

$$
Y \mapsto|Y(\mathbf{0})|+\|Y\|_{\alpha,[0,1]^{k}}
$$

induces a proper norm on $\mathcal{C}^{\alpha}\left([0,1]^{k} ; \mathbb{R}\right)$ and makes it a Banach space.

An important property of the above Hölder semi-norm on $\mathcal{C}^{\alpha}$ is it's ability to scale. The next proposition is a generalization of exercise 4.24 in [13, and will become very useful in later sections. 
Proposition 6. Let $\mathbf{0}<\mathbf{T} \in(0,1)^{k}$ and assume that for all $\rho \in(\mathbf{0}, \mathbf{1}-\mathbf{T})$ the function $f$ satisfies $\|f\|_{\alpha,[\rho, \rho+\mathbf{T}]} \leq M$ (uniformly in $\rho$ ) for a fixed $\alpha \in(0,1)^{k}$, then

$$
\|f\|_{\alpha,[0,1]^{k}} \leq k M 2\left(1 \vee p_{\alpha-1}(\mathbf{0}, \mathbf{T})\right),
$$

where $p_{\gamma}(\mathbf{x}, \mathbf{y})=\sum_{i=1}^{k}\left|y_{i}-x_{i}\right|^{\gamma_{i}}$.

Proof. This is a simple extension of exercise 4.24 in [13].

Earlier we introduced a generalized increment $\square$ "lifting" functions from $C\left([0,1]^{k}\right)$ to $C\left([0,1]^{k} \times[0,1]^{k}\right)$.

We will also need an operator lifting functions from $C\left([0,1]^{k} \times[0,1]^{k}\right)$ to $C\left([0,1]^{k} \times[0,1]^{k} \times[0,1]^{k}\right)$. The idea is familiar to the classical theory of rough paths, and we will give a closer description of this operator in the next definition.

Definition 7. Consider a function $f:[0,1]^{k} \times[0,1]^{k} \rightarrow \mathbb{R}$ and define the operator

$$
V_{i, \mathbf{v}, \mathbf{z}}(\mathbf{x}, \mathbf{y}):=\left(\left(x_{1}, . ., x_{i-1}, v_{i}, x_{i+1}, . ., x_{k}\right),\left(y_{1}, . ., y_{i-1}, z_{i}, y_{i+1}, . ., y_{k}\right)\right)
$$

and let $V_{i, \mathbf{v}, \mathbf{z}} f(\mathbf{x}, \mathbf{y})=f\left(V_{i, \mathbf{v}, \mathbf{z}}(\mathbf{x}, \mathbf{y})\right)$ (notice that $V$ is the same $V$ we have used before for increments, but extended to $\left.[0,1]^{k} \times[0,1]^{k}\right)$. The Chen operator (or delta $(\delta)$ operator)

$$
\delta^{\theta}: C\left([0,1]^{k} \times[0,1]^{k}\right) \rightarrow C\left([0,1]^{k} \times[0,1]^{k} \times[0,1]^{k}\right)
$$

around the points $\mathbf{x}, \mathbf{y} \in[0,1]^{k}$ centered at $\mathbf{z} \in[\mathbf{x}, \mathbf{y}]$ is defined by

$$
\delta_{\mathbf{z}}^{\theta} f(\mathbf{x}, \mathbf{y})=\prod_{i \in \theta}\left(I-\psi_{i}(\mathbf{z})\right) f(\mathbf{x}, \mathbf{y}),
$$

where $\psi_{i}(\mathbf{z})=V_{i, \mathbf{x}, \mathbf{z}}+V_{i, \mathbf{z}, \mathbf{y}}$, and $\theta$ is a set of length $1 \leq l \leq k$ of elements from $\{1, . ., k\}$, i.e.

$$
\theta=\left\{\theta_{1}, . ., \theta_{l}\right\} \in\{1, . ., k\}^{l} .
$$

Also, for some $i \in\{1, . ., k\}$, we can choose $\theta=i$ and we get the special case

$$
\begin{gathered}
\delta_{\mathbf{z}}^{(i)} f(\mathbf{x}, \mathbf{y})=\left(I-V_{i, \mathbf{x}, \mathbf{z}}-V_{i, \mathbf{z}, \mathbf{y}}\right) f(\mathbf{x}, \mathbf{y}) \\
=f(\mathbf{x}, \mathbf{y})-f\left(\mathbf{x},\left(y_{1}, . ., z_{i}, . ., y_{k}\right)\right)-f\left(\left(x_{1}, . ., z_{i}, . ., x_{k}\right), \mathbf{y}\right),
\end{gathered}
$$

which is the delta operator from Rough Path theory with respect to the variables $x_{i}<z_{i}<y_{i}$ on some domain $[0,1]$.

Throughout the article, we will often omit the dependence on $\theta$, when $\theta=\{1, . ., k\}$ which can be considered the canonical choice of $\delta$ for a hyper-cube $[0,1]^{k}$. In other words, we often write $\delta_{\mathbf{z}}:=\delta_{\mathbf{z}}^{\theta}$ when $\theta=\{1, . ., k\}$.

Next we will present a way to decompose the delta operator into a sum of $\delta$ operators in only one variable. As this Lemma is a crucial step in later calculations, we will try to give a very detailed proof, such that the ideas come out clear, and the proof is transparent.

Lemma 8. Let $\delta^{\theta}$ be defined as above with $\theta=\{1, . ., k\}$. Then

$$
\delta^{\theta}=\sum_{\tilde{\theta} \subset \theta}(-1)^{|\tilde{\theta}|-1} \sum_{i \in \tilde{\theta}} \prod_{j \in \tilde{\theta} \backslash\left\{\tilde{\theta}_{1}, \ldots, \tilde{\theta}_{i}\right\}} \psi_{j} \delta^{(i)},
$$

where $|\tilde{\theta}|$ is the number of elements in $\tilde{\theta}$, and where the $\tilde{\theta}$ represents the $2^{k}-1$ possible different subsets of $\theta$ (excluding $\{\emptyset\}$ ), and $\tilde{\theta}_{i}$ is the $i^{\prime}$ th component in $\tilde{\theta}$. 
Proof. Let us write $\delta^{\theta}=\prod_{i \in \theta}\left(I-\psi_{i}\right)$, where $\psi_{i}(\mathbf{z}) f(\mathbf{x}, \mathbf{y})=\left(V_{i, \mathbf{x}, \mathbf{z}}+V_{i, \mathbf{z}, \mathbf{y}}\right) f(\mathbf{x}, \mathbf{y})$ for some $f \in C\left([0,1]^{2 k}\right)$. As the proof is of a more technical nature, we prove the claim first for $k=2$ to get an intuition about the result. Consider $\delta^{(1,2)}$ from Definition 7 and see that

$$
\begin{aligned}
\delta^{(1,2)} & =\delta^{(1)} \delta^{(2)}=\left(I-\psi_{1}\right)\left(I-\psi_{2}\right) \\
& =I-\psi_{1} I-\psi_{2} I+\psi_{1} \psi_{2} .
\end{aligned}
$$

Now add and subtract $I$ to the RHS above, and use that

$$
2\left(I-\psi_{1} \psi_{2}\right)=\delta^{(1)}+\delta^{(2)} \psi_{2}+\delta^{(1)}+\delta^{(2)} \psi_{1}
$$

to find that

$$
\begin{gathered}
\delta^{(1,2)}=\left(I-\psi_{1}\right)+\left(I-\psi_{2}\right)-\left(I-\psi_{1} \psi_{2}\right) \\
=\delta^{(1)}+\delta^{(2)}-\frac{1}{2}\left(\delta^{(1)}+\delta^{(1)} \psi_{2}+\delta^{(2)}+\delta^{(2)} \psi_{1}\right) \\
=\frac{1}{2}\left(\delta^{(1)}+\delta^{(2)}-\delta^{(1)} \psi_{2}-\delta^{(2)} \psi_{1}\right),
\end{gathered}
$$

thus for $k=2$ we can decompose $\delta^{(1,2)}$ into delta operators in each variable, i.e $\delta^{(1)}$ and $\delta^{(2)}$.

Let us also show that it holds for $\delta^{(1,2,3)}$, by using another technique. By direct computations of $\delta^{(1,2,3)}$, it is easily seen that

$$
\begin{gathered}
\delta^{(1,2,3)}=\delta^{(3)} \delta^{(1,2)}=\left(I-\psi_{3}\right)\left(I-\psi_{1} I-\psi_{2} I+\psi_{1} \psi_{2}\right) \\
=I-\psi_{3}-\left(I-\psi_{3}\right) \psi_{1}-\left(I-\psi_{3}\right) \psi_{2}+\left(I-\psi_{3}\right) \psi_{1} \psi_{2} \\
=I-\psi_{3}-\psi_{2}-\psi_{1}+\psi_{1} \psi_{3}+\psi_{3} \psi_{2}+\psi_{1} \psi_{2}-\psi_{1} \psi_{2} \psi_{3} \\
=I+\sum_{\tilde{\theta} \subset\{1,2,3\}} \prod_{j \in \tilde{\theta}}\left(-\psi_{j}\right),
\end{gathered}
$$

where there are $2^{3}-1=7$ possible subsets $\tilde{\theta} \subset \theta=\{1,2,3\}$ i.e. $\tilde{\theta}=\{1\}, \tilde{\theta}=\{2\}, \ldots, \tilde{\theta}=$ $\{1,2,3\}$. Now, there are 3 subsets which consists of an even number of elements in them, i.e $\{1,2\},,\{2,3\},\{1,3\}$ and 4 subsets which consists of an odd number of elements i.e. $\{1\},\{2\},\{3\},\{1,2,3\}$. We can therefore divide the above sum into the negative indices and the positive ones, i.e. write

$$
\sum_{\tilde{\theta} \subset\{1,2,3\}} \prod_{j \in \tilde{\theta}}\left(-\psi_{j}\right)=\sum_{\gamma \subset\{1,2,3\}} \prod_{j \in \gamma} \psi_{j}-\sum_{\gamma^{c} \subset\{1,2,3\}} \prod_{j \in \gamma^{c}} \psi_{j}
$$

where $\gamma$ denotes all subsets of $\{1,2,3\}$ with an even number of elements and $\gamma^{c}$ denotes the subsets of $\{1,2,3\}$ of odd number of elements. Consider now $\sum_{\gamma \subset\{1,2,3\}} \prod_{j \in \gamma} \psi_{j}$ and add $\# \gamma=3$ (where $\# \gamma$ means the number of subsets $\gamma$ ) identity operators and see that

$$
\sum_{\gamma \subset\{1,2,3\}} \prod_{j \in \gamma} \psi_{j}=3 I-\sum_{\gamma \subset\{1,2,3\}}\left(I-\prod_{j \in \gamma} \psi_{j}\right) .
$$

From the simple identity $I-a \tilde{a}=(I-a)+(I-\tilde{a}) a$ we can derive by induction the general identity

$$
\begin{gathered}
I-\prod_{i \in \gamma} a_{i}=\left(I-a_{\gamma_{1}}\right)+\left(I-\prod_{i \in \gamma \backslash\left\{\gamma_{1} \mid\right\}} a_{i}\right) a_{\gamma_{1}} \\
=\left(I-a_{\gamma_{1} \mid}\right)+\left(I-a_{\gamma_{2}}\right) a_{\gamma_{1}}+\left(I-\prod_{i \in \gamma \backslash\left\{\gamma_{1}, \gamma_{2}\right\}} a_{i}\right) a_{\gamma_{2}} a_{\gamma_{1}}, \\
=\sum_{i \in \gamma}\left(I-a_{i}\right) \prod_{j \in \gamma \backslash\left\{\gamma_{1}, \ldots, \gamma_{i}\right\}} a_{j},
\end{gathered}
$$


where $\gamma \backslash\left\{\gamma_{1}, \ldots, \gamma_{i}\right\}=\left\{\gamma_{k} \in \gamma \mid \gamma_{k} \neq \gamma_{1}, \ldots, \gamma_{i}\right\}$. Using this identity for $\sum_{\gamma \subset\{1,2,3\}}\left(I-\prod_{j \in \gamma} \psi_{j}\right)$, we can see that

$$
\begin{gathered}
\sum_{\gamma \subset\{1,2,3\}} \prod_{j \in \gamma} \psi_{j}=3 I-\sum_{\gamma \subset\{1,2,3\}} \sum_{i \in \gamma}\left(I-\psi_{i}\right) \prod_{j \in \gamma \backslash\left\{\gamma_{1}, \ldots, \gamma_{|\gamma|-i}\right\}} \psi_{j} \\
=3 I-\sum_{\gamma \subset\{1,2,3\}} \sum_{i \in \gamma} \delta^{(i)} \prod_{j \in \gamma \backslash\left\{\gamma_{1}, \ldots, \gamma_{i}\right\}} \psi_{j} .
\end{gathered}
$$

By the same method using that there are 4 subsets $\gamma^{c}$, we can see that the negative part

$$
\sum_{\gamma^{c} \subset\{1,2,3\}} \prod_{j \in \gamma^{c}} \psi_{j},=4 I-\sum_{\gamma^{c} \subset\{1,2,3\}} \sum_{i \in \gamma^{c}} \delta^{(i)} \prod_{j \in \gamma^{c} \backslash\left\{\gamma^{c}{ }_{1}, \ldots, \gamma_{i}^{c}\right\}} \psi_{j} .
$$

Combining the two calculations, we get

$$
\begin{aligned}
I+ & \sum_{\gamma^{c} \subset\{1,2,3\}} \sum_{\gamma \subset\{1,2,3\}} \prod_{j \in \gamma} \psi_{j}-\sum_{\gamma^{c} \subset\{1,2,3\}} \prod_{j \in \gamma^{c}} \psi_{j} \\
= & I+3 I-\sum_{\gamma \subset\{1,2,3\}} \sum_{i \in \gamma} \delta^{(i)} \prod_{j \in \gamma \backslash\left\{\gamma_{1}, \ldots, \gamma_{i}\right\}} \psi_{j} \\
& -4 I+\sum_{\gamma^{c} \subset\{1,2,3\}} \sum_{i \in \gamma^{c}} \delta^{(i)} \prod_{j \in \gamma^{c} \backslash\left\{\gamma^{c} 1_{1}, \ldots, \gamma_{i}^{c}\right\}} \psi_{j} \\
= & \sum_{\tilde{\theta} \subset\{1,2,3\}}(-1)^{(|\tilde{\theta}|-1)} \sum_{i \in \tilde{\theta}} \delta^{(i)} \prod_{j \in \tilde{\theta} \backslash\left\{\tilde{\theta}_{1}, \ldots, \tilde{\theta}_{i}\right\}} \psi_{j},
\end{aligned}
$$

where $|\tilde{\theta}|$ denotes the number of elements in $\tilde{\theta}$.

Now we will consider $k \geq 1$ with $\theta=\{1, \ldots, k\}$. By multiplying out the product $\prod_{i \in \theta}\left(I-\psi_{i}\right)$, we obtain that

$$
\prod_{i \in \theta}\left(I-\psi_{i}\right)=I+\sum_{\tilde{\theta}} \prod_{i \in \tilde{\theta}}\left(-\psi_{i}\right) .
$$

Note that the sum $\sum_{\tilde{\theta}} \prod_{i \in \tilde{\theta}}\left(-\psi_{i}\right)$ consists of $2^{k}-1$ components of the form $\prod_{i \in \tilde{\theta}}\left(-\psi_{i}\right)$. Divide this sum into a negative and positive part, i.e

$$
\sum_{\tilde{\theta} \subset \theta} \prod_{i \in \tilde{\theta}}\left(-\psi_{i}\right)=\sum_{\gamma \subset \theta} \prod_{i \in \gamma} \psi_{i}-\sum_{\gamma^{c} \subset \theta} \prod_{i \in \gamma^{c}} \psi_{i}
$$

See that $\# \gamma^{c}=2^{k-1}$, and $\# \gamma=2^{k-1}-1$. Using the Equation 2.1 we find

$$
I-\prod_{i \in \gamma} \psi_{i}=\sum_{i \in \gamma} \delta^{(i)} \prod_{j \in \gamma \backslash\left\{\gamma_{1}, \ldots, \gamma_{i}\right\}} \psi_{j}
$$

we can calculate that

$$
\begin{aligned}
& \sum_{\gamma} \prod_{i \in \gamma} \psi_{i}=\# \gamma I-\sum_{\gamma \subset \theta}\left(I-\prod_{i \in \gamma} \psi_{i}\right) \\
& =\# \gamma I-\sum_{\gamma \subset \theta} \sum_{i \in \gamma} \delta^{(i)} \prod_{j \in \gamma \backslash\left\{\gamma_{1}, \ldots, \gamma_{i}\right\}} \psi_{j} .
\end{aligned}
$$

The same can be found for the negative part,

$$
\sum_{\gamma^{c} \subset \theta} \prod_{i \in \gamma^{c}} \psi_{i}=\# \gamma^{c} I-\sum_{\gamma^{c} \subset \theta} \sum_{i \in \gamma^{c}} \delta^{(i)} \prod_{j \in \gamma^{c} \backslash\left\{\gamma^{c} 1, \ldots, \gamma_{i}^{c}\right\}} \psi_{j}
$$


Combining the two, we can see that

$$
I-\sum_{\tilde{\theta} \subset \theta} \prod_{i \in \tilde{\theta}}\left(-\psi_{i}\right)=\sum_{\tilde{\theta} \subset \theta} \sum_{i \in \tilde{\theta}}(-1)^{|\tilde{\theta}|-1} \delta^{(i)} \prod_{j \in \tilde{\theta} \backslash\left\{\tilde{\theta}_{1}, \ldots, \tilde{\theta}_{i}\right\}} \psi_{j},
$$

where the summation over $\tilde{\theta}$ on the RHS is meant over all possible subsets of $\theta=\{1, \ldots, k\}$, and $(-1)^{|\tilde{\theta}|-1}$ is either +1 or -1 depending on $\tilde{\theta}$. At last we obtain that we can decompose $\delta^{\theta}$ as

$$
\delta^{\theta}=\sum_{\tilde{\theta} \subset \theta} \sum_{i \in \tilde{\theta}}(-1)^{|\tilde{\theta}|-1} \delta^{(i)} \prod_{j \in \tilde{\theta} \backslash\left\{\tilde{\theta}_{1}, \ldots, \tilde{\theta}_{i}\right\}} \psi_{j} .
$$

It is important to note that the product of operators can be written shortly as $\delta^{(i)} \psi_{\tilde{\theta}}^{i}:=$ $\delta^{(i)} \prod_{j \in \tilde{\theta} \backslash\left\{\tilde{\theta}_{1}, \ldots, \tilde{\theta}_{i}\right\}} \psi_{j}$. The operator $\psi_{\tilde{\theta}}^{i}$ is acting on functions $f:[0,1]^{2 k} \rightarrow \mathbb{R}$ and is shuffling around the variable at positions above $i$, and is therefore not "changing" the regularity of the function.

Remark 9. We want to point out that the above delta operator and the one defined by Gubinelli and Chouk [3] are exactly the same when $k=2$, and it can directly be seen as a generalization of their work. Consider a function $f:[0,1]^{2} \times[0,1]^{2} \rightarrow \mathbb{R}$, and write $\psi_{i}(\mathbf{z})=\left(V_{i, \cdot, \mathbf{z}}+V_{i, \mathbf{z}, \cdot}\right)$ for $i=1,2$; the delta operator $\delta$ considered by Gubinelli and Chouk is then given by

$$
\begin{gathered}
\delta_{\mathbf{z}}^{(1,2)}:=\left(I-\psi_{1}(\mathbf{z})\right)\left(I-\psi_{2}(\mathbf{z})\right) \\
=\frac{1}{2}\left(\delta^{(1)}+\delta^{(2)}-\delta^{(1)} \psi_{2}(\mathbf{z})-\delta^{(2)} \psi_{1}(\mathbf{z})\right) .
\end{gathered}
$$

We will be interested in knowing the regularity of the delta operator when acting on functions $\Xi \in \mathcal{C}_{2}^{\alpha}\left([0,1]^{k} ; \mathbb{R}\right)$, and we can use the decomposition of the $\delta$ operator from Lemma 8 to find a criterion for when the inequality

$$
\left|\delta_{\mathbf{z}} \Xi(\mathbf{x}, \mathbf{y})\right| \lesssim q_{\alpha}(\mathbf{x}, \mathbf{y}) p_{\beta-\alpha}(\mathbf{x}, \mathbf{y})
$$

is satisfied for $p_{\beta}(\mathbf{x}, \mathbf{y})=\sum_{i=1}^{k}\left|y_{i}-x_{i}\right|^{\beta_{i}}$ and $q_{\alpha}(\mathbf{x}, \mathbf{y})=\prod_{i=1}^{k}\left|x_{i}-y_{i}\right|^{\alpha_{i}}$.

Lemma 10. Let $\beta \in(1, \infty)^{k}$ and $\theta=\{1, \ldots, k\}$. If $\Xi:[0,1]^{k} \times[0,1]^{k} \rightarrow \mathbb{R}$ and $\Xi$ satisfies

$$
\left|\delta_{\mathbf{z}}^{(i)} \Xi(\mathbf{x}, \mathbf{y})\right| \lesssim q_{\left(\alpha_{1}, \ldots, \beta_{i}, \ldots, \alpha_{k}\right)}(\mathbf{x}, \mathbf{y})
$$

for all $i \in \theta$, then

$$
\left|\delta_{\mathbf{z}}^{\theta} \Xi(\mathbf{x}, \mathbf{y})\right| \lesssim q_{\alpha}(\mathbf{x}, \mathbf{y}) p_{\beta-\alpha}(\mathbf{x}, \mathbf{y}) .
$$

Proof. From Lemma 8 we know that for $\mathbf{z} \in[\mathbf{x}, \mathbf{y}]$

$$
\begin{aligned}
& \left|\delta_{\mathbf{z}}^{\theta} \Xi(\mathbf{x}, \mathbf{y})\right|=\left|\sum_{\tilde{\theta} \subset \theta} \sum_{i \in \tilde{\theta}}(-1)^{|\tilde{\theta}|-1} \prod_{j \in \tilde{\theta} \backslash\left\{\tilde{\theta}_{1}, \ldots, \tilde{\theta}_{i}\right\}} \psi_{j} \delta^{(i)} \Xi(\mathbf{x}, \mathbf{y})\right| \\
& \leq\left\|\delta^{(i)} \Xi\right\|_{\left(\alpha_{1}, . ., \beta_{i}, \ldots, \alpha_{k}\right), \square} \\
& \times \sum_{\tilde{\theta} \subset \theta} \sum_{i \in \tilde{\theta}}(-1)^{|\tilde{\theta}|-1} \prod_{j \in \tilde{\theta} \backslash\left\{\tilde{\theta}_{1}, \ldots, \tilde{\theta}_{i}\right\}} \psi_{j}(\mathbf{z}) q_{\left(\alpha_{1}, . ., \beta_{i}, \ldots, \alpha_{k}\right)}(\mathbf{x}, \mathbf{y}),
\end{aligned}
$$

where

$$
\left\|\delta^{(i)} \Xi\right\|_{\left(\alpha_{1}, ., \beta_{i}, \ldots, \alpha_{k}\right), \square}=\sup _{\mathbf{x} \neq \mathbf{y} \in[0,1]^{k}} \frac{\left|\delta_{\mathbf{z}}^{(i)} \Xi(\mathbf{x}, \mathbf{y})\right|}{q_{\left(\alpha_{1}, . ., \beta_{i}, \ldots, \alpha_{k}\right)}(\mathbf{x}, \mathbf{y})}
$$


Notice that the operator $\prod_{j \in \tilde{\theta} \backslash\left\{\tilde{\theta}_{1}, \ldots, \tilde{\theta}_{|\tilde{\theta}|-i}\right\}} \psi_{j}(\mathbf{z})$ is just changing the variables which is lower than $|\tilde{\theta}|-i$, therefore

$$
\begin{gathered}
\sum_{\tilde{\theta} \subset \theta} \sum_{i \in \tilde{\theta}}(-1)^{|\tilde{\theta}|-1} \prod_{\substack{j \in \tilde{\theta} \backslash\left\{\tilde{\theta}_{1}, \ldots, \tilde{\theta}_{i}\right\} \\
\leq C q_{\left(\alpha_{1}, \ldots, \beta_{i}, \ldots, \alpha_{k}\right)}}} \psi_{j}(\mathbf{x}, \mathbf{y}) q_{\left(\alpha_{1}, . ., \beta_{i}, \ldots, \alpha_{k}\right)}(\mathbf{x}, \mathbf{y}) \\
\leq
\end{gathered}
$$

The next Lemma will prove another important property; remember that $\square$ is the generalized increment operation, and one wants the Chen operator $\delta$ and the increment $\square$ to act in a similar manner as they do in classical rough paths theory, i.e. the $\delta$ operator acting on the generalized increment is 0 .

Lemma 11. If $X \in C\left([0,1]^{k} ; \mathbb{R}\right)$, then for $\theta=\{1, . ., k\}$ we have

$$
\delta_{\mathbf{z}}^{\theta} \square_{\mathbf{x}, \mathbf{y}}^{k} X=0 .
$$

Proof. We write $\mathbf{z}^{\prime}=\left(z_{1}, . ., z_{k-1}\right)$ when $\mathbf{z} \in[0,1]^{k}$ to signify the removal of the $i^{\prime}$ th variable. First, we observe that

$$
\left(I-V_{i, \mathbf{x}, \mathbf{z}}-V_{i, \mathbf{z}, \mathbf{y}}\right) \square_{\mathbf{x}, \mathbf{y}}^{k} X=\square_{\mathbf{x}, \mathbf{y}}^{k} X-\left(\square_{\mathbf{x}, V_{i, \mathbf{z}} \mathbf{y}}^{k} X+\square_{V_{i, \mathbf{z}} \mathbf{x}, \mathbf{y}}^{k} X\right),
$$

we can easily see that for $\theta=\{1, \ldots, k\}$ we have

$$
\begin{gathered}
\square_{\mathbf{x}, V_{i, \mathbf{z}} \mathbf{y}}^{k} X+\square_{V_{i, \mathbf{x}} \mathbf{x}, \mathbf{y}}^{k} X= \\
\prod_{j \in \theta \backslash\{i\}}\left(I-V_{j, \mathbf{x}}\right)\left(\left(I-V_{i, \mathbf{x}}\right) X\left(V_{i, \mathbf{z}} \mathbf{y}\right)+\left(I-V_{i, V_{i, \mathbf{z}} \mathbf{x}}\right) X(\mathbf{y})\right), \\
=\square_{\mathbf{x}, \mathbf{y}}^{k} X
\end{gathered}
$$

and therefore it is clear that

$$
\left(I-V_{i, \mathbf{x}, \mathbf{z}}-V_{i, \mathbf{z}, \mathbf{y}}\right) \square_{\mathbf{x}, \mathbf{y}}^{k} X=0,
$$

and furthermore

$$
\delta_{\mathbf{z}}^{\theta} \square_{\mathbf{x}, \mathbf{y}}^{k} X=\prod_{i \in \theta}\left(I-V_{i, \mathbf{x}, \mathbf{z}}-V_{i, \mathbf{z}, \mathbf{y}}\right) \square_{\mathbf{x}, \mathbf{y}}^{k} X=0 .
$$

In the next section we will see how we can use the $\delta$-operator to prove a generalized sewing Lemma, which then can be used to construct Young integration for fields.

\section{Sewing Lemma and Young integration for FieldS}

We will begin this section with a motivation of the construction of a sewing map for fields on $[0,1]^{k}$. Let us first discuss Riemann sums of functions on hyper-cube domains. We are interested in constructing $k$-fold integrals of the form $\int_{x_{1}}^{y_{1}} \cdots \int_{x_{k}}^{y_{k}}$, and in the case of smooth $f$ and $g$ on $[0,1]^{k}$ this $k$-fold integral can be written as

$$
\begin{aligned}
\int_{x_{1}}^{y_{1}} & \ldots \int_{x_{k}}^{y_{k}} f\left(z_{1}, \ldots, z_{k}\right) \frac{\partial^{k}}{\partial z_{1}, \ldots, \partial z_{k}} g\left(z_{1}, \ldots, z_{k}\right) d z_{1}, \ldots, d z_{k} \\
= & \lim _{\left|\mathcal{P}^{1}\right| \rightarrow 0}, \ldots, \lim _{\left|\mathcal{P}^{k}\right| \rightarrow 0} \sum_{\left[u_{1}, v_{1}\right] \in \mathcal{P}^{1}} \ldots \sum_{\left[u_{1}, v_{1}\right] \in \mathcal{P}^{k}} f\left(u_{1}, \ldots, u_{k}\right) \\
& \times \int_{u_{1}}^{v_{1}} \ldots \int_{u_{k}}^{v_{k}} \frac{\partial^{k}}{\partial z_{1}, \ldots, \partial z_{k}} g\left(z_{1}, \ldots, z_{k}\right) d z_{1}, \ldots, d z_{k} .
\end{aligned}
$$


Each of the partitions $\mathcal{P}^{1}, \ldots, \mathcal{P}^{k}$ are made from a division of the respective intervals $\left[x_{1}, y_{1}\right], \ldots,\left[x_{k}, y_{k}\right]$. Therefore, we could consider grid-like partitions of $[0,1]^{k}$ by a cartesian product of the partitions $\mathcal{P}^{1}, \ldots, \mathcal{P}^{k}$. In this way, we can write a grid like partition $\mathcal{P}[\mathbf{x}, \mathbf{y}]$ of $\prod_{i=1}^{k}\left[x_{i}, y_{i}\right]$ as the product

$$
\mathcal{P}=\prod_{i \in \theta} \mathcal{P}^{i}\left[x_{i}, y_{i}\right]
$$

where $\theta=\{1, . ., k\}$ and where each element $[\mathbf{u}, \mathbf{v}] \in \mathcal{P}[\mathbf{x}, \mathbf{y}]$ is of the form $\prod_{i=1}^{k}\left[u_{i}, v_{i}\right]$ for $\left[u_{i}, v_{i}\right] \in \mathcal{P}^{i}$ for $i \in\{1, \ldots, k\}$. If we also write $\int_{\mathbf{x}}^{\mathbf{y}}=\int_{x_{1}}^{y_{1}} \cdots \int_{x_{k}}^{y_{k}}$ as the integral over a hyper cube $[\mathbf{x}, \mathbf{y}] \subset[0,1]^{k}$, we can write Equation (3.1) in a more compact form

$$
\int_{\mathbf{x}}^{\mathbf{y}} f(\mathbf{z}) D^{\gamma} g(\mathbf{z}) d \mathbf{z}=\lim _{|\mathcal{P}| \rightarrow 0} \sum_{[\mathbf{u}, \mathbf{v}] \in \mathcal{P}} f(\mathbf{u}) \int_{\mathbf{u}}^{\mathbf{v}} D^{\gamma} g(\mathbf{z}) d \mathbf{z}
$$

where $D^{\gamma}=\frac{\partial^{k}}{\partial z_{1}, \ldots, \partial z_{k}}$ is the multi-index notation of partial derivatives with $\gamma=(1, . ., 1)($ i.e. 1 , $k$-times). Moreover, for a hyper cube, $[\mathbf{u}, \mathbf{v}]$ we define by

$$
|[\mathbf{u}, \mathbf{v}]|:=\sup _{j=1, . ., k}\left|v_{j}-u_{j}\right|
$$

Furthermore define the size of the mesh of the partition in the following way

$$
|\mathcal{P}[\mathbf{x}, \mathbf{y}]|:=\sup _{[\mathbf{u}, \mathbf{v}] \in \mathcal{P}[\mathbf{x}, \mathbf{y}]}\{|[\mathbf{u}, \mathbf{v}]|\} .
$$

Remark 12. In [14 the authors discuss variation norms of $2 D$ functions. One of the observations discussed in the paper is that grid-like partitions as defined above is not an exhaustive definition of partitions on hyper cubes, and they show that the study of functions in a grid like setting in some $\rho$-variation norm is equivalent to study $\rho+\epsilon$ variation on general partitions of hyper cubes for some small $\epsilon>0$. From this, we are tempted to believe that the integral we construct in over grid like partitions, may be translated to integrals over general partitions at the cost of some small loss of Hölder regularity. However, we have not checked this but leave this for future investigations.

Before moving to the theorem of the sewing map, we must first give a definition of an appropriate space of abstract "Riemann integrands" satisfying some regularity conditions. With the decomposition of the $\delta$-operator in mind, we give the following definition.

Definition 13. Let $\alpha \in(0,1)^{k}, \beta \in(1, \infty)^{k}$ and $\theta=\{1, . ., k\}$. The space $\mathcal{C}_{2}^{\alpha, \beta, \theta}\left([0,1]^{2 k} ; \mathbb{R}\right)$ is defined by all functions $\Xi:[0,1]^{2 k} \rightarrow \mathbb{R}$, such that

$$
\begin{gathered}
\|\Xi\|_{\alpha, \beta}:=\|\Xi\|_{\alpha}+\left\|\delta^{\theta} \Xi\right\|_{\beta} \\
:=\sup _{\mathbf{x} \neq \mathbf{y} \in[0,1]^{k}} \frac{|\Xi(\mathbf{x}, \mathbf{y})|}{q_{\alpha}(\mathbf{x}, \mathbf{y})}+\sum_{i \in \theta} \sup _{(\mathbf{x}, \mathbf{y}) \in \triangle\left([0,1]^{k}\right) ; z_{i} \in\left[x_{i}, y_{i}\right]} \frac{\left|\delta_{z_{i}}^{(i)} \Xi_{i, \mathbf{x}, \mathbf{y}}(u, v)\right|}{q_{\left(\alpha_{1}, \ldots, \beta_{i}, . ., \alpha_{k}\right)}(\mathbf{x}, \mathbf{y})}<\infty,
\end{gathered}
$$

where $q_{\gamma}(\mathbf{x}, \mathbf{y}):=\prod_{i=1}^{k}\left|y_{i}-x_{i}\right|^{\gamma_{i}}$ for $\gamma \in \mathbb{R}_{+}^{k}$.

By the original ideas of Young, the proof of the sewing Lemma in rough path theory is based on the following property: let $\mathcal{P}[x, y]$ be a partition of $[x, y]$ and for some point $z \in[x, y]$ dividing two sets $\left[z_{-}, z\right],\left[z, z^{+}\right] \in \mathcal{P}[x, y]$ define

$$
\mathcal{P}[x, y] \backslash\{z\}:=\mathcal{P}[x, y] \backslash\left\{\left[z^{-}, z\right],\left[z, z^{+}\right]\right\} \cup\left\{\left[z^{-}, z^{+}\right]\right\} .
$$

We can then study the difference between the two Riemann type sums

$$
\begin{gathered}
\sum_{[u, v] \in \mathcal{P}[x, y] \backslash\{z\}} \Xi(u, v)-\sum_{[u, v] \in \mathcal{P}[x, y]} \Xi(u, v) \\
=\Xi\left(z_{-}, z_{+}\right)-\Xi\left(z_{-}, z\right)-\Xi\left(z, z_{+}\right),
\end{gathered}
$$


where $z_{-}$is the largest point in $\mathcal{P}[x, y]$ smaller than $z$ and $z_{+}$the smallest point larger than $z$. The next lemma can be viewed as a direct extension of the sewing lemma proved by Gubinelli and Chouk for 2D fields in [3].

Lemma 14. (Sewing lemma) Consider a hyper cube $[\mathbf{x}, \mathbf{y}] \subset[0,1]^{k}$ for $\mathbf{x} \neq \mathbf{y}$ and let $\Xi \in$ $\mathcal{C}_{2}^{\alpha, \beta, \theta}\left([0,1]^{k} ; \mathbb{R}\right)$. Then there exists a unique continuous map $\mathcal{I}: \mathcal{C}_{2}^{\alpha, \beta, \theta}\left([0,1]^{k} ; \mathbb{R}\right) \rightarrow \mathcal{C}_{+}^{\alpha}\left([0,1]^{k} ; \mathbb{R}\right)$ such that

$$
\left|\mathcal{I}(\Xi)_{\mathbf{x}, \mathbf{y}}-\Xi(\mathbf{x}, \mathbf{y})\right| \leq C_{\beta, k}\left\|\delta^{\theta} \Xi\right\|_{\beta} q_{\alpha}(\mathbf{x}, \mathbf{y}) p_{\beta-\alpha}(\mathbf{x}, \mathbf{y})
$$

where $p_{\beta}(\mathbf{x}, \mathbf{y})=\sum_{i=1}^{k}\left|y_{i}-x_{i}\right|^{\beta_{i}}$.

Proof. We follow the ideas used in [13] Lemma 4.2, but generalize to fields. As for uniqueness, assume $J$ and $\tilde{J}$ are two candidates for $\mathcal{I}(\Xi)$, then both satisfy the above inequality, which imply that $(J-\tilde{J})(\mathbf{0})=0$ and $(J-\tilde{J})(\mathbf{x}, \mathbf{y}) \lesssim q_{\alpha}(\mathbf{x}, \mathbf{y}) p_{\beta-\alpha}(\mathbf{x}, \mathbf{y})$ for $\beta \in(1, \infty)^{k}$, which tells us that $J-\tilde{J}=0$.

Let $\mathcal{P}^{i}\left[x_{i}, y_{i}\right]$ denote a partition of $\left[x_{i}, y_{i}\right]$ containing $r \geq 2$ number of sets $\forall i \in\{1, \ldots, k\}$. From Lemma 4.2 in [13] we know that there exists a point $z_{i}$ in $\left[x_{i}, y_{i}\right]$ with two corresponding sets $\left[z_{i}^{-}, z_{i}\right],\left[z_{i}, z_{i}^{+}\right] \in \mathcal{P}^{i}\left[x_{i}, y_{i}\right]$ and the following is satisfied

$$
\left|z_{i}^{+}-z_{i}^{-}\right| \leq \frac{2}{r-1}\left|y_{i}-x_{i}\right|
$$

We will continue the proof in two steps, first we show a maximal inequality for partition integrals, and then we show non-dependence of the chosen partition we have used in the integral. The proof is done by induction, i.e we will show that the claim holds for $\theta=\{1,2\}$ and then generalize to $\theta=\{1, \ldots, k\}$.

(1) Let us write $I_{\mathcal{P}^{\theta}}=\sum_{[\mathbf{u}, \mathbf{v}] \in \mathcal{P}^{\theta}}$ as the partition integral (Riemann sum) over some partition defined by $\mathcal{P}^{\theta}[\mathbf{x}, \mathbf{y}]=\prod_{i \in \theta} \mathcal{P}^{i}\left[x_{i}, y_{i}\right]$, i.e for $\Xi \in \mathcal{C}_{2}^{\alpha, \beta, \theta}\left([0,1]^{k} ; \mathbb{R}\right)$, we write

$$
\begin{gathered}
I_{\mathcal{P}^{\theta}[\mathbf{x}, \mathbf{y}]} \Xi:=\sum_{[\mathbf{u}, \mathbf{v}] \in \mathcal{P}^{\theta}} \Xi(\mathbf{u}, \mathbf{v}) \\
=\sum_{\left[u_{1}, v_{1}\right] \in \mathcal{P}\left[x_{1}, y_{1}\right]}, \ldots, \sum_{\left[u_{k}, v_{k}\right] \in \mathcal{P}\left[x_{k}, y_{k}\right]} \Xi(\mathbf{u}, \mathbf{v}) .
\end{gathered}
$$

Using this notation, we know that if $\theta=(1,2, \ldots, k)$ we can write

$$
I_{\mathcal{P}^{\theta}}=I_{\mathcal{P}^{(1)}}, \ldots, I_{\mathcal{P}^{(k)}}=: \prod_{i \in \theta} I_{\mathcal{P}^{(i)}},
$$

where for fixed $\mathbf{x}^{\prime}, \mathbf{y}^{\prime} \in[0,1]^{k-1}$, we have

$$
\begin{gathered}
I_{\mathcal{P}^{(i)}}: \mathcal{C}_{2}^{\alpha_{i}, \beta_{i},(i)}\left([0,1]^{k} ; \mathbb{R}\right) \rightarrow \mathcal{C}^{\alpha} \\
I_{\mathcal{P}^{(i)}\left[x_{i}, y_{i}\right]} \Xi\left(\cdot, \mathbf{x}^{\prime}, \mathbf{y}^{\prime}\right)=\sum_{\left[u_{i}, v_{i}\right] \in \mathcal{P}^{(i)}\left[x_{i}, y_{i}\right]} \Xi\left(u_{i}, v_{i} ; \mathbf{x}^{\prime}, \mathbf{y}^{\prime}\right) .
\end{gathered}
$$

We will mostly write $I_{\mathcal{P}^{(i)}}=I_{\mathcal{P}^{(i)}\left[x_{i}, y_{i}\right] ;[\mathbf{x}, \mathbf{y}]}$ when the set we integrate over is else clear. Moreover, denote by

$$
\mathcal{P}^{(i)}\left[x_{i}, y_{i}\right] \backslash\left\{z_{i}\right\}:=\mathcal{P}^{(i)}\left[x_{i}, y_{i}\right] \backslash\left\{\left[z_{i}^{-}, z_{i}\right],\left[z_{i}, z_{i}^{+}\right]\right\} \cup\left\{\left[z_{i}^{-}, z_{i}^{+}\right]\right\},
$$

and then define $\mathcal{P}^{\theta} \backslash\{\mathbf{z}\}=\prod_{i \in \theta} \mathcal{P}^{(i)}\left[x_{i}, y_{i}\right] \backslash\left\{z_{i}\right\}$ as the partition $\mathcal{P}^{\theta}$ when we remove a point $\mathbf{z} \in[\mathbf{x}, \mathbf{y}]$. From Equation (3.2) we can see that

$$
I_{\mathcal{P}^{(i)}\left[x_{i}, y_{i}\right] \backslash\left\{z_{i}\right\}}=I_{\mathcal{P}^{(i)}\left[x_{i}, y_{i}\right]}+\delta_{z_{i}}^{(i)},
$$


and we can also write

$$
I_{\mathcal{P}^{\theta} \backslash\{\mathbf{z}\}}=\prod_{i \in \theta}\left(I_{\mathcal{P}^{(i)}\left[x_{i}, y_{i}\right]}+\delta_{z_{i}}^{(i)}\right)
$$

Let us first consider $\theta=\{1,2\}$ then it follows from rough path theory (see Lemma 4.2 in [13]) and since $\Xi \in \mathcal{C}_{2}^{\alpha, \beta,\{1,2\}}\left([0,1]^{4} ; \mathbb{R}\right)$, that

$$
\mathcal{I}^{(i)}(\Xi)(\mathbf{x}, \mathbf{y})=\lim _{\left|\mathcal{P}^{(i)}\right| \rightarrow 0} \sum_{\left[u_{i}, v_{i}\right] \in \mathcal{P}^{(i)}\left[x_{i}, y_{i}\right]} \Xi\left(u_{i}, v_{i} ; \mathbf{x}^{\prime}, \mathbf{y}^{\prime}\right)
$$

is well defined for $i \in \theta$. Just as shown above, we can write

$$
\begin{gathered}
\left(I_{\mathcal{P}^{1}\left[x_{1}, y_{1}\right] \backslash\left\{z_{1}\right\}}-I_{\mathcal{P}^{1}}\right)\left(I_{\mathcal{P}^{2}\left[x_{2}, y_{2}\right] \backslash\left\{z_{2}\right\}}-I_{\mathcal{P}^{2}}\right) \Xi \\
=\left(I_{\mathcal{P}^{\theta} \backslash\{\mathbf{z}\}}-I_{\mathcal{P}^{1}} I_{\mathcal{P}^{2} \backslash\left\{z_{2}\right\}}-I_{\mathcal{P}^{2}} I_{\mathcal{P}^{1} \backslash\left\{z_{1}\right\}}+I_{\mathcal{P}^{\theta}}\right) \Xi=\delta_{\mathbf{z}}^{(1,2)} \Xi\left(\mathbf{z}^{-}, \mathbf{z}^{+}\right),
\end{gathered}
$$

and we can see that by Lemma 8 we have the decomposition of $\delta^{(1,2)}$ such that we get

$$
\begin{gathered}
\left|\left(I_{\mathcal{P}^{\theta} \backslash\{\mathbf{z}\}}-I_{\mathcal{P}^{1}} I_{\mathcal{P}^{2} \backslash\left\{z_{2}\right\}}-I_{\mathcal{P}^{2}} I_{\mathcal{P}^{1} \backslash\left\{z_{1}\right\}}+I_{\mathcal{P}^{\theta}}\right) \Xi\right| \\
\leq\left(\left\|\delta^{(1)} \Xi\right\|_{\beta_{1}}+\left\|\delta^{(2)} \Xi\right\|_{\beta_{2}}\right)\left(q_{\beta_{1}, \alpha_{2}}\left(\mathbf{z}^{-}, \mathbf{z}^{+}\right)+q_{\alpha_{1}, \beta_{2}}\left(\mathbf{z}^{-}, \mathbf{z}^{+}\right)\right) \\
=\left(\left\|\delta^{(1)} \Xi\right\|_{\beta_{1}}+\left\|\delta^{(2)} \Xi\right\|_{\beta_{2}}\right) q_{\alpha}\left(\mathbf{z}^{-}, \mathbf{z}^{+}\right) p_{\beta-\alpha}\left(\mathbf{z}^{-}, \mathbf{z}^{+}\right) \\
\leq\left(\left\|\delta^{(1)} \Xi\right\|_{\beta_{1}}+\left\|\delta^{(2)} \Xi\right\|_{\beta_{2}}\right) q_{\alpha}(\mathbf{x}, \mathbf{y}) p_{\beta-\alpha}(\mathbf{x}, \mathbf{y}) \\
\times \sum_{j=1}^{2} \prod_{i=1}^{2}\left(\frac{2}{r-1}\right)^{\alpha_{i}}\left(\frac{2}{r-1}\right)^{\beta_{j}-\alpha_{j}},
\end{gathered}
$$

where we also used inequality (3.3). Furthermore, it is not difficult to see that

$$
\sum_{j=1}^{2} \prod_{i=1}^{2}\left(\frac{2}{r-1}\right)^{\alpha_{i}}\left(\frac{2}{r-1}\right)^{\beta_{j}-\alpha_{j}} \leq \sum_{j=1}^{2}\left(\frac{2}{r-1}\right)^{\beta_{j}} .
$$

By iteration we can find that

$$
\begin{gathered}
\left(I d-I_{\mathcal{P}^{1}}\right)\left(I d-I_{\mathcal{P}^{2}}\right) \Xi(\mathbf{x}, \mathbf{y}) \\
=\left(\sum_{i=1}^{r} I_{\mathcal{P}^{1} \backslash\left\{z_{1}^{1}, \ldots, z_{1}^{i}\right\}}-I_{\mathcal{P}^{1} \backslash\left\{z_{1}^{1}, . ., z_{1}^{i-1}\right\}}\right)\left(\sum_{i=1}^{r} I_{\mathcal{P}^{2} \backslash\left\{z_{2}^{1}, . ., z_{2}^{i}\right\}}-I_{\mathcal{P}^{2} \backslash\left\{z_{2}^{1}, . ., z_{2}^{i-1}\right\}}\right) \Xi \\
=\sum_{i, j=1}^{r}\left(I_{\mathcal{P}^{1} \backslash\left\{z_{1}^{1}, \ldots, z_{1}^{i}\right\}}-I_{\mathcal{P}^{1} \backslash\left\{z_{1}^{1}, \ldots, z_{1}^{i-1}\right\}}\right)\left(I_{\mathcal{P}^{2} \backslash\left\{z_{2}^{1}, . ., z_{2}^{j}\right\}}-I_{\mathcal{P}^{2} \backslash\left\{z_{2}^{1}, \ldots, z_{2}^{j-1}\right\}}\right) \Xi \\
=\sum_{i, j=1}^{r} \delta_{z_{1}^{i}, z_{2}^{j}}^{(1,2)} \Xi\left(z_{1}^{i,-}, z_{2}^{j,-}, z_{1}^{i,+}, z_{2}^{j,+}\right)
\end{gathered}
$$

By the assumption that $\Xi \in \mathcal{C}_{2}^{\alpha, \beta,(1,2)}\left([0,1]^{4} ; \mathbb{R}\right)$, and using inequality (3.3), we can see that

$$
\begin{gathered}
\left|\left(I d-I_{\mathcal{P}^{1}}\right)\left(I d-I_{\mathcal{P}^{2}}\right) \Xi(\mathbf{x}, \mathbf{y})\right| . \\
\lesssim\left(\left\|\delta^{(1)} \Xi\right\|_{\beta_{1}}+\left\|\delta^{(2)} \Xi\right\|_{\beta_{2}}\right) q_{\alpha}(\mathbf{x}, \mathbf{y}) p_{\beta-\alpha}(\mathbf{x}, \mathbf{y}) \sum_{j, i=1}^{r}\left(\left(\frac{2}{i}\right)^{\beta_{1}}+\left(\frac{2}{j}\right)^{\beta_{2}}\right) \\
\leq\left(\left\|\delta^{(1)} \Xi\right\|_{\beta_{1}}+\left\|\delta^{(2)} \Xi\right\|_{\beta_{2}}\right) q_{\alpha}(\mathbf{x}, \mathbf{y}) p_{\beta-\alpha}(\mathbf{x}, \mathbf{y}) \zeta\left(\beta_{1}\right) \zeta\left(\beta_{2}\right),
\end{gathered}
$$

where $\zeta$ is the Riemann-Zeta function, and by $\left\|\delta^{(i)} \Xi\right\|_{\beta_{i}}$ we mean $\left\|\delta^{(i)} \Xi\right\|_{\left(\beta_{i}, \alpha_{j}\right), \square}$ for $i \neq j \in$ $\{1,2\}$. Moreover since $\Xi \in \mathcal{C}_{2}^{\alpha, \beta,(1,2)}\left([0,1]^{4} ; \mathbb{R}\right)$, we know that

$$
\left|I_{\mathcal{P}^{i}} \Xi(\mathbf{x}, \mathbf{y})-\Xi(\mathbf{x}, \mathbf{y})\right| \lesssim\left\|\delta^{(i)} \Xi\right\|_{\beta_{i}} q_{\alpha}(\mathbf{x}, \mathbf{y})\left|x_{i}-y_{i}\right|^{\beta_{i}-\alpha_{i}}
$$


for $i \in \theta$. Also, note the relation

This lets us conclude that

$$
\begin{gathered}
I_{\mathcal{P}^{1,2}}-I d=\left(I d-I_{\mathcal{P}^{1}}\right)\left(I d-I_{\mathcal{P}^{2}}\right) \\
+\left(I_{\mathcal{P}^{1}}-I d\right)+\left(I_{\mathcal{P}^{2}}-I d\right) .
\end{gathered}
$$

$$
\begin{gathered}
\left|I_{\mathcal{P}^{1,2}[\mathbf{x}, \mathbf{y}]} \Xi(\mathbf{x}, \mathbf{y})-\Xi(\mathbf{x}, \mathbf{y})\right| \\
\lesssim\left(\left\|\delta^{(1)} \Xi\right\|_{\beta_{1}}+\left\|\delta^{(2)} \Xi\right\|_{\beta_{2}}\right) q_{\alpha}(\mathbf{x}, \mathbf{y}) p_{\beta-\alpha}(\mathbf{x}, \mathbf{y}),
\end{gathered}
$$

where we have used Next, we will generalize this argument for $k \geq 2$, and $\theta=\{1, \ldots, k\}$. Assume that all integrals up to order $k-1$ exists, and then do the argument by induction. More specifically, we assume that for all subsets $\gamma \subset \theta$ the integrals

$$
\mathcal{I}^{\gamma}(\Xi)(\mathbf{x}, \mathbf{y})=\lim _{\left|\mathcal{P}^{\gamma}\right| \rightarrow 0} \sum_{[\mathbf{u}, \mathbf{v}] \in \mathcal{P}^{\gamma}} \Xi(\mathbf{u}, \mathbf{v} ; \mathbf{x}, \mathbf{y})
$$

where $\Xi(\mathbf{u}, \mathbf{v} ; \mathbf{x}, \mathbf{y})$ denotes the Riemann integrand in the variables $\mathbf{u}, \mathbf{v} \in[0,1]^{\gamma}$ and the are also evaluated in the remaining variables of $\mathbf{x}$ and $\mathbf{y}$. Similarly as for the case when $k=2$, we can use Equation (3.4), we can iterate again, and find

$$
\begin{gathered}
\left|\prod_{i \in \theta}\left(I d-I_{\mathcal{P}^{(i)}}\right) \Xi\right|=\left|\prod_{i \in \theta}\left(\sum_{j=1}^{r} I_{\mathcal{P}^{(i)} \backslash\left\{z_{i}^{1}, ., z_{i}^{j}\right\}}-I_{\mathcal{P}^{(i)} \backslash\left\{z_{i}^{1}, \ldots, z_{i}^{j-1}\right\}}\right)\right| \\
=\left|\sum_{j_{1}, . ., j_{|\theta|}=1}^{r} \prod_{i \in \theta}\left(I_{\mathcal{P}^{(i)} \backslash\left\{z_{i}^{1}, . ., z_{i}^{j_{i}}\right\}}-I_{\mathcal{P}^{(i)} \backslash\left\{z_{i}^{1}, . ., z_{i}^{j_{i}-1}\right\}}\right) \Xi\right| \\
=\left|\sum_{j_{1}, . ., j_{|\theta|}=1}^{r}\left(\prod_{i \in \theta} \delta_{z_{i}^{j_{i}}}^{(i)}\right) \Xi\right| \\
\lesssim\left\|\delta^{\theta} \Xi\right\|_{\beta} \sum_{j_{1}, . ., j_{|\theta|}=1}^{r} q_{\alpha}\left(z_{1}^{j_{1},-}, . . z_{k}^{j_{|\theta|},-} ; z_{1}^{j_{1},+}, . . z_{k}^{j_{|\theta|},+}\right) \\
\times p_{\beta-\alpha}\left(z_{1}^{j_{1},-}, . . z_{k}^{j_{|\theta|},-} ; z_{1}^{j_{1},+}, . . z_{k}^{j_{|\theta|},+}\right),
\end{gathered}
$$

where $|\theta|$ is the number of elements in $\theta$. Using the bounds from Inequality (3.3), we can see that

$$
\left|\prod_{i \in \theta}\left(I d-I_{\mathcal{P}^{(i)}}\right) \Xi\right| \lesssim\left(\sum_{i \in \theta}\left\|\delta^{(i)} \Xi\right\|_{\beta_{i},}\right) q_{\alpha}(\mathbf{x}, \mathbf{y}) p_{\beta-\alpha}(\mathbf{x}, \mathbf{y}) \prod_{i \in \theta} \zeta\left(\beta_{i}\right),
$$

where $\zeta$ is the Riemann-Zeta function which is converging since $\beta_{i}>1$ for all $i=1, . ., k$. Furthermore, for all multi-indices $\gamma \subset \theta=\{1,2, \ldots, k\}$ such that $\gamma$ is a subset of $\theta$ we know by the induction hypothesis that

$$
\left|\left(I_{\mathcal{P}^{\gamma}}-I d\right) \Xi(\mathbf{x}, \mathbf{y})\right| \lesssim\left(\sum_{i \in \gamma}\left\|\delta^{(i)} \Xi\right\|_{\beta_{i}}\right) q_{\alpha}(\mathbf{x}, \mathbf{y}) p_{\beta-\alpha}^{\gamma}(\mathbf{x}, \mathbf{y}),
$$

where $p_{\beta}^{\gamma}(\mathbf{x}, \mathbf{y})=\sum_{i \in \gamma}\left|x_{i}-y_{i}\right|^{\beta_{i}}$, and our goal is to prove that also

$$
\left|\left(I_{\mathcal{P}^{\theta}}-I d\right) \Xi(\mathbf{x}, \mathbf{y})\right| \lesssim\left(\sum_{i \in \theta}\left\|\delta^{(i)} \Xi\right\|_{\beta_{i}}\right) q_{\alpha}(\mathbf{x}, \mathbf{y}) p_{\beta-\alpha}(\mathbf{x}, \mathbf{y}) .
$$

First, notice that for $\theta=\{1, \ldots, k\}$, we can write

$$
\prod_{i \in \theta}\left(I_{\mathcal{P}^{(i)}}-I d\right) \Xi=I_{\mathcal{P}^{\theta}}+(-1)^{|\theta|} I d+\sum_{\gamma \subset \theta}(-1)^{|\gamma|} I_{\mathcal{P}^{\gamma}}
$$


where $|\theta|$ denotes the number of elements in $\theta$. We can see that on the RHS the sum $\sum_{\gamma \subset \theta}(-1)^{|\gamma|} I_{\mathcal{P} \gamma}$ consists of $2^{k}-2$ elements, i.e. there exists $2^{k}-2$ ordered subsets of $\{1,2, . ., k\}$ when we exclude $\{1,2, \ldots, k\}$ and $\{\emptyset\}$. We will distinguish between two scenarios, when $|\theta|$ is even and when it is odd. If $|\theta|$ is odd, then the sum $\sum_{\gamma \subset \theta}(-1)^{|\gamma|} I_{\mathcal{P}^{\gamma}}$ consists of $\left(2^{k}-2\right) / 2$ operators with negative signs, and $\left(2^{k}-2\right) / 2$ with positive sign, and we can add and subtract $\left(2^{k}-2\right) / 2 I d$-operators, to see that

$$
\sum_{\gamma \subset \theta}(-1)^{|\gamma|} I_{\mathcal{P} \gamma} \pm \frac{\left(2^{k}-2\right)}{2} I d=\sum_{\gamma \subset \theta}(-1)^{|\gamma|+1}\left(I_{\mathcal{P} \gamma}-I d\right) .
$$

We can rearrange the above relation, and together with Equation (3.5) we obtain that

$$
\left|\left(I_{\mathcal{P}^{\theta}}-I d\right) \Xi\right| \lesssim \sum_{\gamma \subset \theta}\left|\left(I_{\mathcal{P}^{\gamma}}-I d\right) \Xi\right|+\left|\prod_{i \in \theta}\left(I_{\mathcal{P}^{(i)}}-I d\right) \Xi\right| .
$$

Combining with our previous estimates, we can see that

$$
\begin{gathered}
\left|\left(I_{\mathcal{P}^{\theta}}-I d\right) \Xi(\mathbf{x}, \mathbf{y})\right| \\
\lesssim \sum_{\gamma \subset \theta}\left(\left(\sum_{i \in \gamma}\left\|\delta^{(i)} \Xi\right\|_{\beta_{i}}\right) p_{\beta-\alpha}^{\gamma}(\mathbf{x}, \mathbf{y})+\left(\sum_{i \in \theta}\left\|\delta^{(i)} \Xi\right\|_{\beta_{i}}\right) p_{\beta-\alpha}(\mathbf{x}, \mathbf{y})\right) q_{\alpha}(\mathbf{x}, \mathbf{y}) \\
\lesssim 2^{k}\left(\sum_{i \in \theta}\left\|\delta^{(i)} \Xi\right\|_{\beta_{i}}\right) p_{\beta-\alpha}(\mathbf{x}, \mathbf{y}) q_{\alpha}(\mathbf{x}, \mathbf{y}) .
\end{gathered}
$$

On the other hand, if $|\theta|$ is even, then the sum $\sum_{\gamma \subset \theta}(-1)^{|\gamma|} I_{\mathcal{P}^{\gamma}}$ consists of two more negative operators. Rewriting Equation (3.5) we have the equality

$$
I_{\mathcal{P}^{\theta}}=\prod_{i \in \theta}\left(I_{\mathcal{P}^{(i)}}-I d\right) \Xi-\left(I d+\sum_{\gamma \subset \theta}(-1)^{|\gamma|} I_{\mathcal{P}^{\gamma}}\right),
$$

Now, add and subtract $2^{k-1} I d$ operators, and we obtain

$$
I_{\mathcal{P}^{\theta}}=\prod_{i \in \theta}\left(I_{\mathcal{P}^{(i)}}-I d\right) \Xi-I d-\sum_{\gamma \subset \theta}(-1)^{|\gamma|}\left(I_{\mathcal{P}^{\gamma}}-I d\right)+2 I d,
$$

and rewriting this expression and applying it to the integrand $\Xi$, we conclude just as in the case of odd $|\theta|$ that

$$
\left|\left(I_{\mathcal{P}^{\theta}}-I d\right) \Xi\right| \lesssim 2^{k}\left(\sum_{i \in \theta}\left\|\delta^{(i)} \Xi\right\|_{\beta_{i}}\right) p_{\beta-\alpha}(\mathbf{x}, \mathbf{y}) q_{\alpha}(\mathbf{x}, \mathbf{y}) .
$$

This lets us conclude that the above inequality holds for any $\theta=\{1, \ldots, k\}$.

Also note that the above inequality is independent of which points that divides up the partition $\mathcal{P}[\mathbf{x}, \mathbf{y}]$. Therefore, let $\tilde{\mathcal{P}}[\mathbf{x}, \mathbf{y}]$ denote any grid-like partition, and we obtain a maximal inequality,

$$
\left|\Xi(\mathbf{x}, \mathbf{y})-I_{\tilde{\mathcal{P}}[\mathbf{x}, \mathbf{y}]} \Xi\right| \lesssim 2^{k}\left(\sum_{i \in \theta}\left\|\delta^{(i)} \Xi\right\|_{\beta_{i}}\right) p_{\beta-\alpha}(\mathbf{x}, \mathbf{y}) q_{\alpha}(\mathbf{x}, \mathbf{y})
$$

(2) Next, we will show that we have convergence of the integral regardless of the chosen grid-like partition. That is, we will show that the $\operatorname{limit} \lim _{|\mathcal{P}[\mathbf{x}, \mathbf{y}]| \rightarrow 0} I_{\mathcal{P}[\mathbf{x}, \mathbf{y}]} \Xi$ is independent of the partition $\mathcal{P}[\mathbf{x}, \mathbf{y}]$. This is equivalent to showing

$$
\sup _{|\mathcal{P}[\mathbf{x}, \mathbf{y}]| \vee\left|\mathcal{P}^{\prime}[\mathbf{x}, \mathbf{y}]\right| \leq \epsilon}\left|I_{\mathcal{P}^{\prime}[\mathbf{x}, \mathbf{y}]} \Xi-I_{\mathcal{P}[\mathbf{x}, \mathbf{y}]} \Xi\right| \rightarrow 0 \text { as } \epsilon \rightarrow 0 .
$$

Assume without loss of generality that $\left|\mathcal{P}^{\prime}[\mathbf{x}, \mathbf{y}]\right| \vee|\mathcal{P}[\mathbf{x}, \mathbf{y}]|=|\mathcal{P}[\mathbf{x}, \mathbf{y}]|$, i.e. $\mathcal{P}^{\prime}[\mathbf{x}, \mathbf{y}]$ refines $\mathcal{P}[\mathbf{x}, \mathbf{y}]$ in the sense that for all $i \in\{1, . ., k\}$ we have $\left|\mathcal{P}^{\prime,(i)}\left[x_{i}, y_{i}\right]\right| \vee\left|\mathcal{P}^{(i)}\left[x_{i}, y_{i}\right]\right|=\left|\mathcal{P}^{(i)}\left[x_{i}, y_{i}\right]\right|$. Using that 
$\mathcal{P}^{\prime}[\mathbf{x}, \mathbf{y}]=\prod_{i \in \theta} \bigcup_{\left[u_{i}, v_{i}\right] \in \mathcal{P}^{(i)}\left[x_{i}, y_{i}\right]} \mathcal{P}^{\prime,(i)}\left[x_{i}, y_{i}\right] \cap\left[u_{i}, v_{i}\right]$, we can use a similar trick as we did before and write

$$
\begin{aligned}
& I_{\mathcal{P}^{\prime}}=\prod_{i \in \theta} I_{\bigcup_{\left[u_{i}, v_{i}\right] \in \mathcal{P}^{(i)}\left[x_{i}, y_{i}\right]} \mathcal{P}^{\prime,(i)}\left[x_{i}, y_{i}\right] \cap\left[u_{i}, v_{i}\right]} \\
& =\prod_{i \in \theta} I_{\left[u_{i}, v_{i}\right] \in \mathcal{P}^{(i)}\left[x_{i}, y_{i}\right]} I_{\mathcal{P}^{\prime},(i)}\left[x_{i}, y_{i}\right] \cap\left[u_{i}, v_{i}\right] \\
& =\prod_{i \in \theta} I_{\bigcup_{\left[u_{i}, v_{i}\right] \in \mathcal{P}^{(i)}\left[x_{i}, y_{i}\right]}} \prod_{i \in \theta} I_{\mathcal{P}^{\prime},(i)}\left[x_{i}, y_{i}\right] \cap\left[u_{i}, v_{i}\right] .
\end{aligned}
$$

Therefore, we can compare

$$
\begin{gathered}
\left|\left(I_{\mathcal{P}}-I_{\mathcal{P}^{\prime}}\right) \Xi\right| \\
\left.\leq \prod_{i \in \theta} I_{\bigcup_{\left[u_{i}, v_{i}\right] \in \mathcal{P}^{(i)}\left[x_{i}, y_{i}\right]} \mid \prod_{i \in \theta}\left(I-I_{\mathcal{P}^{\prime},(i)}\left[x_{i}, y_{i}\right] \cap\left[u_{i}, v_{i}\right]\right.}\right) \Xi \mid,
\end{gathered}
$$

by the maximal inequality showed above, we have

$$
\begin{gathered}
\prod_{i \in \theta}\left(I-I_{\mathcal{P}^{\prime},(i)}\left[x_{i}, y_{i}\right] \cap\left[u_{i}, v_{i}\right]\right. \\
\vdots \\
\lesssim C_{\beta} 2^{k}\left(\sum_{i \in \theta}\left\|\delta^{(i)} \Xi\right\|_{\beta_{i}}\right)\left(\prod_{i \in \theta} I_{\mathcal{P}^{(i)}\left[x_{i}, y_{i}\right]}\right) p_{\beta-\alpha}(\mathbf{u}, \mathbf{v}) q_{\alpha}(\mathbf{u}, \mathbf{v})
\end{gathered}
$$

where

$$
\begin{gathered}
\prod_{i \in \theta} I_{\mathcal{P}^{(i)}\left[x_{i}, y_{i}\right]} p_{\beta-\alpha}(\mathbf{u}, \mathbf{v}) q_{\alpha}(\mathbf{u}, \mathbf{v})=\sum_{[\mathbf{u}, \mathbf{v}] \in \mathcal{P}[\mathbf{x}, \mathbf{y}]} p_{\beta-\alpha}(\mathbf{u}, \mathbf{v}) q_{\alpha}(\mathbf{u}, \mathbf{v}) . \\
\leq \sum_{\left[u_{1}, v_{1}\right] \in \mathcal{P}^{(1)}} \ldots \sum_{\left[u_{k}, v_{k}\right] \in \mathcal{P}^{(k)}} \sum_{i=1}^{k}\left|v_{i}-u_{i}\right|^{\beta_{i}} .
\end{gathered}
$$

Note that for $\beta \in(1, \infty)$, we have $\sum_{[\mathbf{u}, \mathbf{v}] \in \mathcal{P}[\mathbf{x}, \mathbf{y}]} \sum_{i=1}^{k}\left|v_{i}-u_{i}\right|^{\beta_{i}}=\sum_{i=1}^{k} \epsilon^{\beta_{i}-1}\left|x_{i}-y_{i}\right|$,and it follows that

which proves our claim, and concludes the proof.

$$
\sup _{|\mathcal{P}[\mathbf{x}, \mathbf{y}]| \vee\left|\mathcal{P}^{\prime}[\mathbf{x}, \mathbf{y}]\right| \leq \epsilon}\left|\int_{\mathcal{P}^{\prime}[\mathbf{x}, \mathbf{y}]} \Xi-\int_{\mathcal{P}[\mathbf{x}, \mathbf{y}]} \Xi\right| \lesssim \epsilon^{\inf \beta_{i}-1},
$$

Next we will show how $\delta$ operates on functions of the form $Y(\mathbf{x}) \square_{\mathbf{x}, \mathbf{y}}^{k} X$ for some $\mathbf{x}, \mathbf{y} \in[0,1]^{k}$ which plays a crucial role in the construction of the Young integral (as we can consider this the local approximation of the Young integral operator).

Lemma 15. Let $\theta=\{1, . ., k\}$ and $\Xi(\mathbf{x}, \mathbf{y}):=Y(\mathbf{x}) \square_{\mathbf{x}, \mathbf{y}}^{k} X$ for some $X, Y \in C\left([0,1]^{k}\right)$. For some $\mathbf{z} \in[\mathbf{x}, \mathbf{y}]$ we have for all $j \in \theta$

$$
\delta_{\mathbf{z}}^{(j)} \Xi(\mathbf{x}, \mathbf{y})=-\left(Y\left(V_{j, \mathbf{z}} \mathbf{x}\right)-Y(\mathbf{x})\right) \square_{V_{j, \mathbf{z}} \mathbf{x}, \mathbf{y}}^{k} X .
$$

Proof. Using the commuting property $V_{i, \mathbf{x}} V_{j, \mathbf{x}}=V_{j, \mathbf{x}} V_{i, \mathbf{x}}$ for all $i, j \in\{1, . ., k\}$ we can see that

$$
\begin{gathered}
\square_{\mathbf{x}, \mathbf{y}}^{k} X-\square_{\mathbf{x},\left(y_{1}, . ., z_{j}, . ., y_{k}\right)}^{k} X=\prod_{i=1}^{j-1}\left(I-V_{i,\left(y_{1}, . ., z_{j}, . ., y_{k}\right)}\right) \times \prod_{i=j+1}^{k}\left(I-V_{i,\left(y_{1}, . ., z_{j}, . ., y_{k}\right)}\right) \\
\times\left(X\left(x_{1}, . ., z_{j}, . ., x_{k}\right)-X\left(x_{1}, . ., y_{j}, . ., x_{k}\right)\right) \\
=\prod_{i=1}^{j-1}\left(I-V_{i, \mathbf{y}}\right) \times \prod_{i=j+1}^{k}\left(I-V_{i, \mathbf{y}}\right)\left(I-V_{j, \mathbf{y}}\right) X\left(x_{1}, . ., z_{j}, . ., x_{k}\right) \\
=\square_{\left(x_{1}, . ., z_{j}, . ., x_{k}\right), \mathbf{y}}^{k} X
\end{gathered}
$$


and we can see that

$$
\begin{aligned}
\delta_{\mathbf{z}}^{(j)} Y(\mathbf{x}) \square_{\mathbf{x}, \mathbf{y}}^{k} X= & Y(\mathbf{x}) \square_{\mathbf{x}, \mathbf{y}}^{k} X-Y(\mathbf{x}) \square_{\mathbf{x}, V_{j, \mathbf{z}} \mathbf{y}}^{k} X-Y\left(V_{j, \mathbf{z}} \mathbf{x}\right) \square_{V_{j, \mathbf{z}} \mathbf{x}, \mathbf{y}}^{k} X \\
& =-\left(Y\left(V_{j, \mathbf{z}} \mathbf{x}\right)-Y(\mathbf{x})\right) \square_{V_{j, \mathbf{z}} \mathbf{x}, \mathbf{y}}^{k} X .
\end{aligned}
$$

With the above Lemma we are now ready to present the an integration theorem for Young fields on hyper-cubes $[0,1]^{k}$.

Theorem 16. Let $Y \in \mathcal{C}^{\alpha}\left([0,1]^{k} ; \mathbb{R}\right)$ and $X \in \mathcal{C}^{\beta}\left([0,1]^{k} ; \mathbb{R}\right)$ for two multi-indices $\alpha \in(0,1)^{k}$ and $\beta \in(0,1)^{k}$ with the property that

$$
\alpha_{i}+\beta_{i}>1 \forall i \in\{1, . ., k\},
$$

and assume $[\mathbf{x}, \mathbf{y}]$ is non-degenerate. Then there exists a $C_{\alpha, \beta, k}>0$ such that

$$
\left|\int_{\mathbf{x}}^{\mathbf{y}} Y(\mathbf{z}) X(d \mathbf{z})-Y(\mathbf{x}) \square_{\mathbf{x}, \mathbf{y}}^{k} X\right| \leq C_{\alpha, \beta, k}\|Y\|_{\alpha,+}\|X\|_{\beta, \square} q_{\beta}(\mathbf{x}, \mathbf{y}) p_{\alpha}(\mathbf{x}, \mathbf{y})
$$

with $p_{\gamma}(\mathbf{x}, \mathbf{y})=\sum_{i \in \theta}\left|x_{i}-y_{i}\right|^{\gamma_{i}}$ and $q_{\gamma}(\mathbf{x}, \mathbf{y})=\prod_{i \in \theta}\left|x_{i}-y_{i}\right|^{\gamma_{i}}$. Moreover, it follows that

$$
\mathbf{x} \mapsto \int_{\mathbf{0}}^{\mathbf{x}} Y(\mathbf{z}) X(d \mathbf{z}) \in \mathcal{C}^{\beta} .
$$

Proof. Set $\Xi(\mathbf{x}, \mathbf{y}):=Y(\mathbf{x}) \square_{\mathbf{x}, \mathbf{y}} X$ then we must show that for $\theta=\{1, . ., k\}$, we have

$$
\left\|\delta^{\theta} \Xi\right\|_{\alpha+\beta}<\infty
$$

From Lemma 15, we have for $\mathbf{z} \in[\mathbf{x}, \mathbf{y}]$,

$$
\delta_{\mathbf{z}}^{(j)} \Xi(\mathbf{x}, \mathbf{y})=-\left(Y\left(V_{j, \mathbf{z}} \mathbf{x}\right)-Y(\mathbf{x})\right) \square_{V_{j, \mathbf{z}} \mathbf{x}, \mathbf{y}}^{k} X .
$$

Therefore, using the regularity of $Y \in \mathcal{C}_{+}^{\alpha}$ and $X \in \mathcal{C}_{\square}^{\beta}$ it is clear that for $\theta=\{1, . ., k\}$, we have

$$
\begin{gathered}
\left\|\delta^{\theta} \Xi\right\|_{\alpha, \beta} \leq C_{\beta, \alpha} \sum_{i=1}^{k} \sup _{\left(x_{1}, . ., x_{i-1}, x_{i+1}, . ., x_{k}\right) \in[0,1]^{k-1}}\left\|Y_{i, \mathbf{x}}\right\|_{\alpha_{i}}\|X\|_{\beta, \square}\left|y_{i}-x_{i}\right|^{\alpha_{i}} q_{\beta}(\mathbf{x}, \mathbf{y}) \\
\leq C_{\alpha, \beta}\|Y\|_{\alpha,+}\|X\|_{\beta, \square} q_{\beta}(\mathbf{x}, \mathbf{y}) p_{\alpha}(\mathbf{x}, \mathbf{y}) .
\end{gathered}
$$

Now the inequality and the construction of the integral follows from Lemma 14.

For the second claim that

$$
\mathbf{x} \mapsto \int_{\mathbf{0}}^{\mathbf{x}} Y(\mathbf{z}) X(d \mathbf{z}) \in \mathcal{C}^{\beta},
$$

we note that for $\mathbf{x} \neq \mathbf{y} \in[0,1]^{k}$ (i.e $x_{i} \neq y_{i} \forall i \in\{1, \ldots, k\}$ ) we have $\int_{0}^{\mathbf{x}}=\int_{0}^{x_{1}} \cdots \int_{0}^{x_{k}}$, and by the linearity of the integrals we get

$$
\int_{0}^{y_{1}} \cdots \int_{0}^{y_{k-1}} \int_{0}^{y_{k}}-\int_{0}^{y_{1}} \cdots \int_{0}^{y_{k-1}} \int_{0}^{x_{k}}=\int_{0}^{y_{1}} \cdots \int_{0}^{y_{k-1}} \int_{x_{k}}^{y_{k}} .
$$

Therefore, we can use an iterative argument to see that

$$
\begin{gathered}
\square_{\mathbf{x}, \mathbf{y}}^{k} \int_{\mathbf{0}}^{\cdot} Y(\mathbf{z}) X(\mathbf{d} \mathbf{z})=\square_{\mathbf{x}^{\prime}, \mathbf{y}^{\prime}}^{k-1} \int_{\mathbf{0}}^{\left(\cdot, y_{k}\right)} Y(\mathbf{z}) X(\mathbf{d} \mathbf{z})-\square_{\mathbf{x}^{\prime}, \mathbf{y}^{\prime}}^{k-1} \int_{\mathbf{0}}^{\left(\cdot, x_{k}\right)} Y(\mathbf{z}) X(\mathbf{d} \mathbf{z}) \\
=\square_{\mathbf{x}^{\prime}, \mathbf{y}^{\prime}}^{k-1} \int_{\left(\cdot, x_{k}\right)}^{\left(\cdot, y_{k}\right)} Y(\mathbf{z}) X(\mathbf{d z}) \\
\vdots \\
=\int_{\mathbf{x}}^{\mathbf{y}} Y(\mathbf{z}) X(d \mathbf{z}) .
\end{gathered}
$$


From this we can see that for $Z(\mathbf{x}):=\int_{0}^{\mathbf{x}} Y(\mathbf{z}) X(d \mathbf{z})$, we have for all $i \in\{1, \ldots, k\}$

$$
\begin{gathered}
\left|Z_{i, \mathbf{x}}\left(v_{i}\right)-Z_{i, \mathbf{x}}\left(u_{i}\right)\right|=\left|\square_{V_{i, \mathbf{v}} \mathbf{0}, V_{i, \mathbf{u}} \mathbf{x}} Z\right| \\
\lesssim\left(\left(|Y(\mathbf{0})|+C_{\alpha, \beta}\|Y\|_{\alpha,+}\right)\|X\|_{\beta, \square}\right)\left|v_{i}-u_{i}\right|^{\alpha_{i}},
\end{gathered}
$$

and together with the first estimate, we obtain our claim.

The above Theorem gives us a construction of the Young integral for fields based on the generalized increment. We can also see that the Hölder continuity of the integral is of order $\beta$ inherited by the driving noise $X \in \mathcal{C}^{\beta}$. This can easily be seen by adding and subtracting $Y(\mathbf{x}) \square_{\mathbf{x}, \mathbf{y}}^{k} X$ and using the above Theorem, we get

$$
\left|\int_{\mathbf{x}}^{\mathbf{y}} Y(\mathbf{z}) X(\mathbf{d z})\right| \leq\left(|Y(\mathbf{0})|+C\|Y\|_{\alpha} p_{\alpha}(\mathbf{x}, \mathbf{y})\right)\|X\|_{\beta, \square} q_{\beta}(\mathbf{x}, \mathbf{y}) .
$$

\section{Differential equations driven by Young Fields}

Using the results established in the previous sections we will now show existence and uniqueness of solutions to differential equations driven by Hölder fields on the form

$$
Y(\mathbf{x})=\xi(\mathbf{x})+\int_{\mathbf{0}}^{\mathbf{x}} f(Y(\mathbf{z})) X(d \mathbf{z}),
$$

for some sufficiently smooth function $f$, and $\xi, X \in \mathcal{C}^{\alpha}\left([0,1]^{k} ; \mathbb{R}\right)$ with $\alpha \in\left(\frac{1}{2}, 1\right)^{k}$. We will construct solutions as a fixed point iteration in the space $\mathcal{C}^{\alpha}$,

The function $\xi(\mathbf{x})$ can be thought of as the boundary behavior of $Y$. Indeed, note that the integral $\int_{\mathbf{0}}^{\mathbf{x}}$ is 0 even when one of the variables $x_{i}$ in $\mathbf{x}$ is 0 , and therefore when one (or more) variable(s) is 0 (i.e. we are one the boundary of the hyper-cube $[0,1]^{k}$ ), then $Y$ is completely determined by $\xi$. When $k=1$, it would be sufficient to describe the initial value of the solution (as a point in the 1 -dim domain). However, when considering hyper-cubes, it is usually necessary to provide more information about the behavior of the solution on the boundary, and therefore $\xi$ is meant to capture just this behavior.

Theorem 17. Let $X \in \mathcal{C}^{\alpha}\left([0,1]^{k} ; \mathbb{R}\right)$ with $\alpha \in\left(\frac{1}{2}, 1\right)^{k}, \xi_{0} \in \mathcal{C}^{\alpha}\left([0,1]^{k} ; \mathbb{R}\right)$ with $\xi_{0}(\mathbf{0}) \in \mathbb{R}$ and $f \in C_{b}^{2}(\mathbb{R} ; \mathbb{R})$. There exists a unique solution in $\mathcal{C}^{\alpha}\left([0,1]^{k} ; \mathbb{R}\right)$ to the rough field equation

$$
Y(\mathbf{x})=\xi_{0}(\mathbf{x})+\int_{\mathbf{0}}^{\mathbf{x}} f(Y(\mathbf{z})) X(d \mathbf{z}),
$$

where the integral is understood as a rough field integral in terms of Theorem 16.

Proof. We will use techniques from [13, based on proving a unique fixed point of a Picard type solution map in the space $\mathcal{C}^{\beta}\left([0,1]^{k} ; \mathbb{R}\right)$ for $\beta \in\left(\frac{1}{2}, \alpha\right) \subset(0,1)^{k}$. After this, we show that the solution is actually in the the space $\mathcal{C}^{\alpha}$ as a consequence of the regularity of the driving field $X \in \mathcal{C}^{\alpha}\left([0,1]^{k}\right)$. The proof will be done in two parts and is based on the behavior of a linear solution map on the space $\mathcal{C}^{\beta}([\rho, \rho+\mathbf{T}] ; \mathbb{R})$ for some $\mathbf{T}>\mathbf{0}$ and $\rho \in[\mathbf{0}, \mathbf{1}-\mathbf{T}]$. When considering the space $\mathcal{C}^{\beta}([\rho, \rho+\mathbf{T}] ; \mathbb{R})$ it is with respect to $X$ also restricted to this space, i.e. $X \in \mathcal{C}^{\beta}([\rho, \rho+\mathbf{T}] ; \mathbb{R})$.

First we will show that the solution map is invariant on a properly constructed unit ball in $\mathcal{C}^{\beta}([\rho, \rho+\mathbf{T}] ; \mathbb{R})$, and then we will show that it is a contraction mapping on $\mathcal{C}^{\beta}([\rho, \rho+\mathbf{T}] ; \mathbb{R})$. Both of these properties will depend on the choice of a small size of $\mathbf{T}>\mathbf{0}$. Therefore, our second objective is to extend the solution from the hyper cube $[\rho, \rho+\mathbf{T}]$ to all of $[0,1]^{k}$ by gluing together the solutions on all the sets $[\rho, \rho+\mathbf{T}]$. 
Consider now some fixed $\mathbf{T}>\mathbf{0}, \rho \in[\mathbf{0}, \mathbf{1}-\mathbf{T}]$, and a hyper cube $[\rho, \rho+\mathbf{T}]$. We can reformulate equation (4.1) to make the proof a bit simpler and consider $\xi_{\rho} \in \mathcal{C}^{\beta}([\rho, \rho+\mathbf{T}])$ which satisfy for some $M \in \mathbb{R}$

$$
\sup _{\rho \in[\mathbf{0}, \mathbf{1}-\mathbf{T}]}\left\|\xi_{\rho}\right\|_{\beta ;[\rho, \rho+\mathbf{T}]} \leq M\left\|\xi_{0}\right\|_{\beta ;[0,1]^{k}} .
$$

Define a solution mapping

$$
\begin{gathered}
\mathcal{V}_{\mathbf{T}}^{\rho}: \mathcal{C}^{\beta}([\rho, \rho+\mathbf{T}]) \rightarrow \mathcal{C}^{\beta}([\rho, \rho+\mathbf{T}]) \\
Y \mapsto \mathcal{V}_{\mathbf{T}}^{\rho}(Y):=\xi_{\rho}(\cdot)+\int_{\rho} f(Y(\mathbf{z})) X(d \mathbf{z}),
\end{gathered}
$$

where the integrals runs from $\rho$ as the initial value of the set $[\rho, \rho+\mathbf{T}]$. The fact that this mapping indeed is in $\mathcal{C}_{\square}^{\beta}$ is a consequence of Theorem 16, and the fact that for $i \in\{1, \ldots, k\}$

$$
\begin{gathered}
\left|f\left(Y_{i, \mathbf{x}}\left(y_{i}\right)+\xi_{\rho, i, \mathbf{x}}\left(y_{i}\right)\right)-f\left(Y_{i, \mathbf{x}}\left(x_{i}\right)+\xi_{\rho, i, \mathbf{x}}\left(x_{i}\right)\right)\right| \\
\lesssim|f|_{C_{b}^{1}}\left(Y_{i, \mathbf{x}}\left(y_{i}\right)-Y_{i, \mathbf{x}}\left(x_{i}\right)+\xi_{\rho, i, \mathbf{x}}\left(y_{i}\right)-\xi_{\rho, i, \mathbf{x}}\left(x_{i}\right)\right) \mid \\
\lesssim|f|_{C_{b}^{1}}\left(\|Y\|_{\beta}+\left\|\xi_{\rho}\right\|_{\beta}\right)\left|y_{i}-x_{i}\right|^{\beta_{i}} .
\end{gathered}
$$

Without loss of generality, in the rest of the proof we consider a subspace of $\mathcal{C}^{\beta}([\rho, \rho+\mathbf{T}])$ of all fields $Y$ which satisfy $Y(\rho)=\xi_{\rho}(\rho)$. Next, we construct a unit ball in $\mathcal{C}^{\beta}$ centered at 0 defined by

$$
\mathcal{B}_{\rho, \mathbf{T}}:=\left\{Y \in \mathcal{C}^{\beta}([\rho, \rho+\mathbf{T}]) \mid\|Y\|_{\beta} \leq 1\right\},
$$

and we want to show that for $Y \in \mathcal{B}_{\rho, \mathbf{T}}$ we can choose $\mathbf{T}$ such that $\mathcal{V}_{\mathbf{T}}(Y) \in \mathcal{B}_{\rho, \mathbf{T}}$. However, from Theorem 16 together with Equation (4.2), we know

$$
\begin{gathered}
\left|\square_{\mathbf{x}, \mathbf{y}} \xi_{\rho}+\int_{\mathbf{x}}^{\mathbf{y}} f(Y(\mathbf{z})) X(d \mathbf{z})\right| \\
\leq\left(\left\|\xi_{\rho}\right\|_{\beta, \square}+\left(|Y|_{\infty}+\|Y\|_{\beta}\right)\|X\|_{\beta, \square} p_{\beta}(\mathbf{x}, \mathbf{y})\right) q_{\beta}(\mathbf{x}, \mathbf{y})
\end{gathered}
$$

Therefore we see that

$$
\left\|\mathcal{V}_{\mathbf{T}}(Y)\right\|_{\beta} \lesssim\left(\left\|\xi_{\rho}\right\|_{\alpha}+\left(\left|\xi_{\mathbf{0}}\right|+\|Y\|_{\beta}\right)\|X\|_{\alpha, \square}\right) p_{\alpha-\beta}(\mathbf{0}, \mathbf{T}),
$$

where we have used that $\|X\|_{\beta, \square \leq}\|X\|_{\alpha, \square} q_{\alpha-\beta}(\mathbf{0}, \mathbf{T})$ and that

$$
\left\|\mathcal{V}_{\mathbf{T}}(Y)\right\|_{\beta}=\sum_{i=1}^{k} \sup _{\mathbf{x} \in[\rho, \rho+\mathbf{T}]}\left\|\mathcal{V}_{\mathbf{T}}(Y)_{i, \mathbf{x}}\right\|_{\beta_{i}}+\left\|\mathcal{V}_{\mathbf{T}}(Y)\right\|_{\beta, \square}
$$

then for

$$
\left|\mathcal{V}_{\mathbf{T}}(Y)_{i, \mathbf{x}}(v)-\mathcal{V}_{\mathbf{T}}(Y)_{i, \mathbf{x}}(u)\right|=\left|\xi_{\rho, i, \mathbf{x}}(v)-\xi_{\rho, i, \mathbf{x}}(u)+\int_{V_{i \mathbf{u}} \mathbf{0}}^{V_{i, \mathbf{v}} \mathbf{x}} f(Y(\mathbf{z})) X(d \mathbf{z})\right|,
$$

we can also use inequality (4.3). From this estimate, it is clear that we can choose $\mathbf{T}=\mathbf{T}_{0}$ small enough such that $p_{\alpha-\beta}\left(\mathbf{0}, \mathbf{T}_{0}\right)$ (notice that $\mathbf{T}_{0}$ is independent of $\rho$ ) gets sufficiently small to obtain the requirement

$$
\left\|\mathcal{V}_{\mathbf{T}_{0}}(Y)\right\|_{\beta ;\left[\rho, \rho+\mathbf{T}_{0}\right]} \leq 1
$$

and we can conclude that $\mathcal{V}_{\mathbf{T}_{0}}^{\rho}$ leaves $\mathcal{B}_{\rho, \mathbf{T}_{\mathbf{0}}}$ invariant.

Next, we will show that $\mathcal{V}_{\mathbf{T}}$ is a contraction mapping on $\mathcal{C}^{\beta}([\rho, \rho+\mathbf{T}])$. We can easily see that for two elements $Y, Z \in \mathcal{C}^{\beta}$, both with boundary given by $\xi_{\rho} \in \mathcal{C}^{\alpha}$ and satisfying

$$
\|Y\|_{\beta},\|Z\|_{\beta} \leq M
$$

we use Theorem 16 to see that

$$
\begin{gathered}
\left\|\mathcal{V}_{\mathbf{T}}(Y)-\mathcal{V}_{\mathbf{T}}(Z)\right\|_{\beta, \square} \\
=\left\|\int_{\rho} f(Y(\mathbf{z})) X(d \mathbf{z})-\int_{\rho} f(Z(\mathbf{z})) X(d \mathbf{z})\right\|_{\beta, \square} \\
\leq C\|f(Y)-f(Z)\|_{\beta,+}\|X\|_{\alpha, \square} q_{\alpha-\beta}(\mathbf{0}, \mathbf{T}) .
\end{gathered}
$$


We must therefore show that

$$
\|f(Y)-f(Z)\|_{\beta,+} \lesssim\|Y-Z\|_{\beta},
$$

but this is straight forward, using Lemma 8.2 in [13, we know that in the

$$
\left\|f\left(Y_{i, \mathbf{x}}\right)-f\left(Z_{i, \mathbf{x}}\right)\right\|_{\beta_{i}} \leq C_{\beta, M}\|f\|_{C_{b}^{2}}\left(\left\|Y_{i, \mathbf{x}}-Z_{i, \mathbf{x}}\right\|_{\beta_{i}}\right),
$$

and we can conclude that Equation (4.5) holds. Therefore,

$$
\left\|\mathcal{V}_{\mathbf{T}}(Y)-\mathcal{V}_{\mathbf{T}}(Z)\right\|_{\beta, \square} \leq C_{\beta, M}\|f\|_{C_{b}^{2}}\|Y-Z\|_{\beta}\|X\|_{\alpha, \square} q_{\alpha-\beta}(\mathbf{0}, \mathbf{T}),
$$

and we can find that

$$
\left\|\mathcal{V}_{\mathbf{T}}(Y)-\mathcal{V}_{\mathbf{T}}(Z)\right\|_{\beta} \leq \tilde{C}_{\beta, M}\|f\|_{C_{b}^{2}}\|Y-Z\|_{\beta}\|X\|_{\alpha, \square} p_{\alpha-\beta}(\mathbf{0}, \mathbf{T}) .
$$

Again, it is clear that we can choose $\mathbf{T}$ small enough such that

$$
\left\|\mathcal{V}_{\mathbf{T}}(Y)-\mathcal{V}_{\mathbf{T}}(Z)\right\|_{\beta} \leq q\|Y-Z\|_{\beta}
$$

for $q \in(0,1)$. We can conclude that $\mathcal{V}_{\mathbf{T}_{0}}$ admits a unique fixed point in $\mathcal{B}_{\rho, \mathbf{T}_{0}}$ which is the solution to equation (4.1). We can now consider Equation (4.4), and choose $\tilde{\mathbf{T}}_{0}$ small, such that $\left(1-\|X\|_{\alpha, \square} p_{\alpha-\beta}\left(\mathbf{0}, \tilde{\mathbf{T}}_{0}\right)\right)>0$, and see that the solution $Y \in \mathcal{C}^{\beta}$ satisfy

$$
\|Y\|_{\beta}\left(1-\|X\|_{\alpha, \square} p_{\alpha-\beta}(\mathbf{0}, \mathbf{T})\right) \leq C_{X}\left(\left\|\xi_{\rho}\right\|_{\alpha}+\left|\xi_{\mathbf{0}}\right|\right),
$$

and we see that on $\left[\rho, \rho+\tilde{\mathbf{T}}_{0} \vee \mathbf{T}_{0}\right]$ we have

$$
\|Y\|_{\beta} \leq C_{X}\left(\left\|\xi_{\rho}\right\|_{\alpha}+\left|\xi_{\mathbf{0}}\right|\right) \leq M C_{X}\left(\left\|\xi_{0}\right\|_{\alpha}+\left|\xi_{\mathbf{0}}\right|\right),
$$

where we used the assumption that $\left\|\xi_{\rho}\right\|_{\alpha} \leq M\left\|\xi_{0}\right\|_{\alpha}$ uniformly in $\rho$.

The second part of the proof is to extend the solution from $\mathcal{C}^{\beta}([\rho, \rho+\mathbf{T}])$ to $\mathcal{C}^{\beta}\left([0,1]^{k}\right)$. First notice that the interval $[\rho, \rho+\mathbf{T}]$ was created for some arbitrary point $\rho \in[\mathbf{0}, \mathbf{1}-\mathbf{T}]$, and specially there exists a solution to equation (4.1) on $[\mathbf{0}, \mathbf{T}]$. From classical theory of differential equations, proofs of existence an uniqueness of solutions on an interval $[0,1]$ is based on first proving existence on $[0, T]$ for some small $T>0$ and then iterate this solution on $[0, T],[T, 2 T] \ldots,[k T, 1]$ by choosing the initial value of the solution on each interval to be the terminal value on the interval prior. However, when we work on hyper cubes, in general, we need to give information about the solution on the boundary of the hyper cube we work on to be able to make sure that the solution is of sufficient continuity when gluing together the cubes in the end. Notice that the hyper cube $[\mathbf{0}, \mathbf{T}]$ is one of $2^{k}$ subsets of $[\mathbf{0}, 2 \mathbf{T}]$ divided in $\mathbf{T}$. When we want to iterate the solution from $[\mathbf{0}, \mathbf{T}]$ to one of these $2^{k}$ adjoining hyper cubes, we must specify the choice of $\xi$ to make sure that the adjoining boundaries of these hyper cubes have the same values. We know that the $2^{k}$ hyper cube subsets of $[\mathbf{0}, 2 \mathbf{T}]$ divided in $\mathbf{T}$ are of the form

$$
[\rho, \rho+\mathbf{T}]=\left[0, T_{1}\right] \times\left[T_{2}, 2 T_{2}\right] \times . . \times\left[T_{k-1}, 2 T_{k-1}\right] \times\left[0, T_{k}\right],
$$

for $\rho=\left(0, T_{2}, . ., T_{k-1}, 0\right)$. First, we want to extend the solution from $[\mathbf{0}, \mathbf{T}]$ to $\left[\gamma^{(1)}, \gamma^{(1)}+\mathbf{T}\right]$ for $\gamma^{(1)}=\left(0, . ., 0, T_{i}, 0, . ., 0\right)$ for some $0 \leq i \leq k$ (the number 1 in the superscript signifies that it is one non-zero entry in $\gamma^{(1)}$ ) and we must consider the equation

$$
Y(\mathbf{x})=\xi_{0}(\mathbf{x})+\int_{\mathbf{0}}^{\mathbf{x}} f(Y(\mathbf{z})) X(d \mathbf{z}), \mathbf{x} \in\left[\gamma^{(1)}, \gamma^{(1)}+\mathbf{T}\right]
$$

and create a suitable solution map with respect to this equation, similar to what we have done earlier for general $[\rho, \rho+\mathbf{T}]$. Note that the integral $\int_{\mathbf{0}}^{\mathbf{x}}$ can be split in the $i-\mathrm{Th}$ component of an integral $\int_{0}^{x_{i}}=\int_{0}^{T_{i}}+\int_{T_{i}}^{x_{i}}$, and obtain the equation

$$
Y^{\left[\gamma^{(1)}, \gamma^{(1)}+\mathbf{T}\right]}(\mathbf{x})=\xi_{0}(\mathbf{x})+\int_{0}^{x_{1}} \cdots \int_{0}^{T_{i}} \cdots \int_{0}^{x_{k}} f(Y(\mathbf{z})) X(d \mathbf{z})
$$




$$
\begin{gathered}
+\int_{0}^{x_{1}} \ldots \int_{T_{i}}^{x_{i}} \ldots \int_{0}^{x_{k}} f(Y(\mathbf{z})) X(d \mathbf{z}) \\
=\left(\xi_{0}(\mathbf{x})-\xi_{0}\left(x_{1}, . ., T_{i}, . ., x_{k}\right)\right)+Y^{[[\mathbf{0}, \mathbf{T}]}\left(x_{1}, . ., T_{i}, . ., x_{k}\right)+\int_{\gamma^{(1)}}^{\mathbf{x}} f(Y(\mathbf{z})) X(d \mathbf{z})
\end{gathered}
$$

where $Y^{[[\mathbf{0}, \mathbf{T}]}$ is the solution to equation (4.1) on $[\mathbf{0}, \mathbf{T}]$. Define

$$
\xi_{\gamma^{(1)}}(\mathbf{x}):=\left(\xi_{0}(\mathbf{x})-\xi_{0}\left(V_{i, \gamma^{(1)}} \mathbf{x}\right)\right)+Y^{\mid[\mathbf{0}, \mathbf{T}]}\left(V_{i, \gamma^{(1)}} \mathbf{x}\right) .
$$

To prove existence and uniqueness, of $Y^{\mid\left[\gamma^{(1)}, \gamma^{(1)}+\mathbf{T}\right]}(\mathbf{x})$ in the above equation, we know it is sufficient to prove that $\xi_{\gamma^{(1)}} \in \mathcal{C}^{\alpha}\left(\left[\gamma^{(1)}, \gamma^{(1)}+\mathbf{T}\right] ; \mathbb{R}\right)$, and that $\left\|\xi_{\gamma^{(1)}}\right\|_{\beta} \leq M\left\|\xi_{0}\right\|_{\beta}$, and then apply the general result for existence and uniqueness on general domains $\left[\gamma^{(1)}, \gamma^{(1)}+\mathbf{T}\right]$ that we obtained in the beginning of this proof. We can see that $\xi_{0} \in \mathcal{C}^{\beta}\left(\left[\gamma^{(1)}, \gamma^{(1)}+\mathbf{T}\right] ; \mathbb{R}\right)$ as it is in $\mathcal{C}^{\beta}\left([0,1]^{k} ; \mathbb{R}\right)$ and it is evident that

$$
\mathbf{x} \mapsto Y^{\mid[\mathbf{0}, \mathbf{T}]}\left(V_{i, \gamma^{(1)}} \mathbf{x}\right) \in \mathcal{C}^{\beta}\left(\left[\gamma^{(1)}, \gamma^{(1)}+\mathbf{T}\right] ; \mathbb{R}\right) .
$$

Indeed, notice that $\mathbf{x} \mapsto Y^{\mid[\mathbf{0}, \mathbf{T}]}\left(V_{i, \gamma^{(1)}} \mathbf{x}\right)$ is constant in variable $i$. Moreover, we have that for all $1 \leq i \leq k$

$$
\begin{aligned}
& \left\|Y^{\mid[\mathbf{0}, \mathbf{T}]}\left(V_{i, \gamma^{(1)}} \cdot\right)\right\|_{\beta ;\left[\gamma^{(1)}, \gamma^{(1)}+\mathbf{T}\right]} \\
& \quad \leq\left\|Y^{[\mathbf{0}, \mathbf{T}]}(\cdot)\right\|_{\beta ;[\mathbf{0}, \mathbf{T}]} \\
& \leq M\left\|\xi_{0}, \xi_{0}^{\prime}\right\|_{\beta ;[0,1]^{k}}
\end{aligned}
$$

where the last bound is obtained from Equation (4.7). It follows that there exists a solution

$$
\mathbf{x} \mapsto Y^{l\left[\gamma^{(1)}, \gamma^{(1)}+\mathbf{T}\right]}(\mathbf{x})
$$

on $\left[\gamma^{(1)}, \gamma^{(1)}+\mathbf{T}\right]$ which is connected to the solution $Y^{\mid[\mathbf{0}, \mathbf{T}]}$ on the boundary. The result holds for all $\gamma^{(1)}=\left(0, . ., T_{i}, . ., 0\right)$, i.e. all $i \in\{1, . ., k\}$.

More generally, consider the operator $D_{\mathbf{z}}^{\theta}=\prod_{i \in \theta}\left(V_{i, \mathbf{x}, \mathbf{z}}+V_{i, \mathbf{z}, \mathbf{y}}\right)$ which is dividing the interval $[\mathbf{x}, \mathbf{y}]$ in $\mathbf{z}$ and considers a linear evaluation of a function on this division. Consider the interval $[\mathbf{0}, \mathbf{x}]$ for $\mathbf{x} \in\left[\gamma^{(1)}, \gamma^{(1)}+\mathbf{T}\right]$, the point $\gamma^{(1)}$ divides $[\mathbf{0}, \mathbf{x}]$ into two hyper cubes, as seen in Equation (4.8). From now on we will use the division of hyper cubes induced by $D$. Next we assume that $\gamma^{(2)}=\left(0, . .0, T_{i}, 0, . ., 0, T_{j}, 0, . ., 0\right)$ i.e. $\gamma^{(2)}$ contains 2 non zero entries with $T_{i}$ and $T_{j}$ for $i \neq j$. We then want to construct the solution to,

$$
Y(\mathbf{x})=\xi_{0}(\mathbf{x})+\int_{\mathbf{0}}^{\mathbf{x}} f(Y(\mathbf{z})) X(d \mathbf{z}), \mathbf{x} \in\left[\gamma^{(2)}, \gamma^{(2)}+\mathbf{T}\right]
$$

again using $D_{\mathbf{z}}^{\theta}$ with $\theta=(i, j)$ and the linearity of the integral, we write

$$
\begin{gathered}
Y(\mathbf{x})=\xi_{0}(\mathbf{x})+D_{\gamma^{(2)}}^{\theta}\left(\int_{\mathbf{0}}^{\mathbf{x}} f(Y(\mathbf{z})) X(d \mathbf{z})\right) \\
=\xi_{0}(\mathbf{x})+\left(V_{i, \mathbf{0}, \gamma^{(2)}}+V_{i, \gamma^{(2)}, \mathbf{x}}\right)\left(V_{j, \mathbf{0}, \gamma^{(2)}}+V_{j, \gamma^{(2)}, \mathbf{x}}\right)\left(\int_{\mathbf{0}}^{\mathbf{x}} f(Y(\mathbf{z})) X(d \mathbf{z})\right)
\end{gathered}
$$

The integral $\int_{\mathbf{0}}^{\mathbf{x}}$ is divided into 4 parts, one of them is depending on the solution $Y^{[[\mathbf{0}, \mathbf{T}]}\left(\cdot, . ., T_{i}, . ., T_{j}, . ., \cdot\right)$

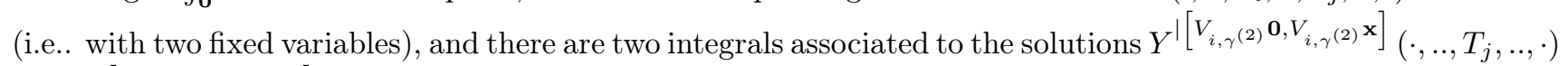
and $Y^{\mid\left[V_{j, \gamma^{(2)}} \mathbf{0}, V_{j, \gamma^{(2)} \mathbf{x}}\right]}\left(\cdot, . ., T_{i}, . ., \cdot\right)$ both already constructed as solutions in the prior step (showing solutions on

$$
\left[\gamma^{(1)}, \gamma^{(1)}+\mathbf{T}\right]=\left[0, T_{1}\right] \times \ldots \times\left[T_{i}, 2 T_{i}\right] \times \ldots \times\left[0, T_{k}\right]
$$

for all $1 \leq i \leq k$. ). The forth is the integral with respect to the the hyper cube $\left[\gamma^{(2)}, \gamma^{(2)}+\mathbf{T}\right]$. Simi-

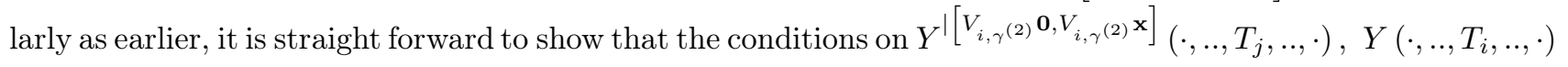


and $Y^{\mid[\mathbf{0}, \mathbf{T}]}\left(\cdot, . ., T_{i}, . ., T_{j}, . ., \cdot\right)$ are fulfilled to conclude that there exists a unique solution to Equation (4.1) on $\left[\gamma^{(2)}, \gamma^{(2)}+\mathbf{T}\right]$ when $\gamma^{(2)}$ contains two non-zero entries of the form $\left(T_{i}, T_{j}\right)$, and we know that the solution on this domain is identical on its boundary as the solutions on the domains we have constructed prior.

By induction principles, we assume there exists unique solutions with boundaries identical to the prior constructed solutions on all intervals of the form $\left[\gamma^{(i-1)}, \gamma^{(i-1)}+\mathbf{T}\right]$ where $\gamma^{(i-1)}$ contains $i-1$ non zero entries each chosen in order from $\left(T_{1}, . ., T_{k}\right)$. That is, at position $n$ in $\gamma^{(i-1)}$ it is either 0 or $T_{n}$ for any $1 \leq n \leq k$. Then we want to prove that the solution exists for any hyper cube $\left[\gamma^{(i)}, \gamma^{(i)}+\mathbf{T}\right]$ where $\gamma^{(i)}$ consist of $i$ ordered and unique non-zero entries as described for $\gamma^{(i-1)}$. Consider $\theta \in\{1, . ., k\}^{i}$ of $i$ ordered components, i.e. if $i \leq j$ then $\theta$ contains no entries $(. ., j ., ., i, .$.$) .$ For $\mathbf{x} \in\left[\gamma^{(i)}, \gamma^{(i)}+\mathbf{T}\right]$ we want to divide $[\mathbf{0}, \mathbf{x}]$ in $\gamma^{(i)}$ according to $\theta$ in the sense that

$$
D_{\gamma^{(i)}}^{\theta}\left(\int_{\mathbf{0}}^{\mathbf{x}} f(Y(\mathbf{z})) X(d \mathbf{z})\right)=\prod_{l \in \theta}\left(V_{l, \mathbf{x}, \mathbf{z}}+V_{l, \mathbf{z}, \mathbf{y}}\right) \int_{\mathbf{0}}^{\mathbf{x}} f(Y(\mathbf{z})) X(d \mathbf{z}),
$$

which would yield a sum of $2^{i}$ integrals. One of these integrals is an integral over $\left[\gamma^{(i)}, \gamma^{(i)}+\mathbf{T}\right]$,and another is over $\left[\mathbf{0}, \gamma^{(i)}\right]$, which would represent the solution on $[\mathbf{0}, \mathbf{T}]$ with $i$ fixed variables $\left\{T_{l}\right\}_{l \in \theta}$ - All other integrals represent solutions on domains $\left[\gamma^{(n)}, \gamma^{(n)}+\mathbf{T}\right]$ for $1<n<i$ with $i-n$ fixed variables. All of these solutions on domains on the form $\left[\gamma^{(n)}, \gamma^{(n)}+\mathbf{T}\right]$ for $1 \leq n<i$ are already proven to exist uniquely (by induction assumption ) and are connected on each of their boundaries, and can therefore be collected in a term called $\xi_{\gamma^{(i)}}$. Similar to what we have shown earlier for $\gamma^{(1)}$ and $\gamma^{(2)}$, it follows that $\xi_{\gamma^{(i)}} \in \mathcal{C}^{\beta}\left(\left[\gamma^{(i)}, \gamma^{(i)}+\mathbf{T}\right] ; \mathbb{R}\right)$, and we can show that $\left\|\xi_{\gamma^{(i)}}\right\|_{\beta} \leq M\left\|\xi_{0}\right\|_{\beta}$. Therefore, we conclude that there exists a unique solution to Equation (4.1) on any domain $\left[\gamma^{(i)}, \gamma^{(i)}+\mathbf{T}\right]$ for all $1 \leq i \leq k$. In particular there exists solutions on all hyper-cubes on the form

$$
\left[0, T_{1}\right] \times \ldots \times\left[T_{j}, 2 T_{j}\right] \times \ldots \times\left[T_{k}, 2 T_{k}\right],
$$

and all the solutions is connected on its boundary to the other solutions. This leads to the conclusion that there exists a unique solution to Equation (4.1) on all $2^{k}$ hyper cubes which is subsets of $[\mathbf{0 , 2 T}]$ divided in $\mathbf{T}$, and by Proposition 6, we conclude that there exists a unique solution to Equation (4.1) on $[\mathbf{0}, 2 \mathbf{T}]$. We then iterate this procedure to obtain existence and uniqueness on all of $[0,1]^{k}$.

Corollary 18. Let $X, \tilde{X} \in \mathcal{C}^{\alpha}\left([0,1]^{k} ; \mathbb{R}\right)$ be two Hölder fields of regularity $\alpha \in\left(\frac{1}{2}, 1\right)^{k}$. Assume $Y, \tilde{Y} \in \mathcal{C}^{\alpha}$ are two solutions to the correpsonding equations

$$
\begin{gathered}
Y(\mathbf{x})=\xi(\mathbf{x})+\int_{\mathbf{0}}^{\mathbf{x}} f(Y(\mathbf{z})) X(d \mathbf{z}) \\
\tilde{Y}(\mathbf{x})=\tilde{\xi}(\mathbf{x})+\int_{\mathbf{0}}^{\mathbf{x}} f(\tilde{Y}(\mathbf{z})) \tilde{X}(d \mathbf{z})
\end{gathered}
$$

for $\xi, \tilde{\xi} \in \mathcal{C}^{\alpha}$ and $f \in C_{b}^{2}(\mathbb{R} ; \mathbb{R})$. Choose $M \in \mathbb{R}$ such that

$$
\|X\|_{\alpha} \vee\|\tilde{X}\|_{\alpha} \vee\|Y\|_{\alpha} \vee\|\tilde{Y}\|_{\alpha} \leq M
$$

Then for all $\beta<\alpha \in\left(\frac{1}{2}, 1\right)^{k}$ we have

$$
\|Y-\tilde{Y}\|_{\beta} \leq C_{M, f}\left(|\xi(\mathbf{0})-\tilde{\xi}(\mathbf{0})|+\|\xi-\tilde{\xi}\|_{\beta}+\|X-\tilde{X}\|_{\beta, \square}\right) .
$$

Proof. Again consider the solution on a hyper-cube $[\rho, \rho+\mathbf{T}]$. Note that $Y$ is the fixed point of a solution map

$$
Z(\mathbf{x}):=\mathcal{V}_{\mathbf{T}}(Y)(\mathbf{x})=\left(\xi(\mathbf{x})+\int_{0}^{\mathbf{x}} f(Y(\mathbf{z})) X(d \mathbf{z})\right)
$$


similar to that constructed in the proof of Theorem [17, and we have the same for $\tilde{Y}$ and $\tilde{\xi}$ in $\mathcal{C}^{\alpha}$ Moreover, it is clear that

$$
\|Y-\tilde{Y}\|_{\beta} \leq\|\xi-\tilde{\xi}\|_{\beta}+\left\|\int_{0}^{\cdot} f(Y(\mathbf{z})) X(d \mathbf{z})-\int_{0}^{\cdot} f(\tilde{Y}(\mathbf{z})) \tilde{X}(d \mathbf{z})\right\|_{\beta} .
$$

From Theorem 16] we have that

$$
\begin{gathered}
\left|\square_{\mathbf{x}, \mathbf{y}} \int_{0}^{\cdot} f(Y(\mathbf{z})) X(d \mathbf{z})-\square_{\mathbf{x}, \mathbf{y}} \int_{0}^{\cdot} f(\tilde{Y}(\mathbf{z})) \tilde{X}(d \mathbf{z})\right| \\
\leq\left|f(Y(\mathbf{x})) \square_{\mathbf{x}, \mathbf{y}} X-f(\tilde{Y}(\mathbf{x})) \square_{\mathbf{x}, \mathbf{y}} \tilde{X}\right| \\
+\sum_{i=1}^{k}\left\|\delta^{(i)}(f(Y) \square X-f(Y) \square \tilde{X})\right\|_{2 \beta} q_{\beta}(\mathbf{x}, \mathbf{y}) p_{\beta}(\mathbf{x}, \mathbf{y}) .
\end{gathered}
$$

using techniques as in the proof of Theorem [16 one can see that

$$
\begin{gathered}
\left\|\delta^{(i)}(f(Y) \square X-f(Y) \square \tilde{X})\right\|_{2 \beta} \\
\leq C\left(\|f(Y)-f(\tilde{Y})\|_{\beta,+}\|X\|_{\beta, \square}+\|f(\tilde{Y})\|_{\beta,+}\|X-\tilde{X}\|_{\beta, \square}\right),
\end{gathered}
$$

Furthermore, we can see that

$$
\begin{gathered}
f(Y(\mathbf{x})) \square_{\mathbf{x}, \mathbf{y}} X-f(\tilde{Y}(\mathbf{x})) \square_{\mathbf{x}, \mathbf{y}} \tilde{X} \\
=(f(Y(\mathbf{x}))-f(\tilde{Y}(\mathbf{x}))) \square_{\mathbf{x}, \mathbf{y}} X+f(\tilde{Y}(\mathbf{x}))\left(\square_{\mathbf{x}, \mathbf{y}} X-\square_{\mathbf{x}, \mathbf{y}} \tilde{X}\right) .
\end{gathered}
$$

and we know that

$$
\|f(Y)-f(\tilde{Y})\|_{\infty} \leq|f(Y(\mathbf{0}))-f(\tilde{Y}(\mathbf{0}))|+\|f(Y)-f(\tilde{Y})\|_{\beta},
$$

and using Equation (4.5) we can see

$$
\|f(Y)-f(\tilde{Y})\|_{\infty} \leq C_{\beta, M}\|f\|_{C_{b}^{2}}\left(|\xi(\mathbf{0})-\tilde{\xi}(\mathbf{0})|+\|f\|_{C_{b}^{2}}\|Y-\tilde{Y}\|_{\beta}\right) .
$$

Also, it is clear that

$$
\left|\square_{\mathbf{x}, \mathbf{y}} X-\square_{\mathbf{x}, \mathbf{y}} \tilde{X}\right| \leq\|X-\tilde{X}\|_{\beta, \square} q_{\beta}(\mathbf{x}, \mathbf{y})
$$

and therefore

$$
\begin{gathered}
\|Y-\tilde{Y}\|_{\beta} \\
\leq C_{M, f}\left(|\xi(\mathbf{0})-\tilde{\xi}(\mathbf{0})|+\|\xi-\tilde{\xi}\|_{\beta}+\|X-\tilde{X}\|_{\beta, \square}+\|Y-\tilde{Y}\|_{\beta} p_{\alpha-\beta}(\mathbf{0}, \mathbf{T})\right) .
\end{gathered}
$$

From and we again choose $\mathbf{T}=\frac{1}{2}$ such that for all hyper-cubes $[\rho, \rho+\mathbf{T}]$

$$
\|Y-\tilde{Y}\|_{\beta ;[\rho, \rho+\mathbf{T}]} \leq C_{M, f} 2\left(|\xi(\mathbf{0})-\tilde{\xi}(\mathbf{0})|+\|\xi-\tilde{\xi}\|_{\beta}+\|X-\tilde{X}\|_{\beta, \square}\right),
$$

then by the scalability of Hölder norms from Lemma [6 we obtain the desired result. 


\section{REFERENCES}

[1] Lawrence Evans. Partial differental equations. American mathematical society, second edition edition, 2010.

[2] Almut A. D. Veraart Fred E. Benth, Ole B. Nielssen. Ambit processes and stochastic partial differential equations. Advanced Mathematical Methods for Finance, pages 35-74, 2011.

[3] M. Gubinelli and K. Chouk. Rough sheets. Arxiv 1406 7748v1, July 2014.

[4] Massimiliano Gubinelli. Controlling rough paths. arxiv 0306433, 2003.

[5] Martin Hairer. Solving the kpz equation. Annals of Mathematics, 178:559-664, 2013.

[6] Martin Hairer. A theory of regularity structures. Inventiones mathematicae, Volume 198:269-504, November 2014.

[7] Yaozhong $\mathrm{Hu}$ and Khoa Le. A multiparamulti Garsia Rodemich Rumsey Inequality and some applications. Stochastic processes and their applications, 2013.

[8] Terry Lyons. Differeintial Equations driven by Rough Signals. Revista Matem $\tilde{A}_{j}$ tica Iberoamericana, 14, 1998.

[9] Samy Tindel Massimiliano Gubinelli. Rough Evolution Equations. Annals of Probability, 2010.

[10] P. Imkeller N. Perkowski, M. Gubinelli. Paracontrolled distributions and singular pdes. Arxiv 1210.2684, 2012.

[11] Fred E. Benth Ole Barndorff-Nielsen and Almut Veraart. Ambit Stochastics. To Appear, 2018.

[12] A. Gulisashvili P. Friz, B. Gess and S. Riedel. The Jain Monrad Criterion For Rough Paths and Applications to Random Fourier Series and Non Markovian Hormander Theory. Annals of Probability, Vol 44 No. 1, 2016.

[13] M Hairer P Friz. A Course on Rough Paths with an introduction to regularity structures. Springer, 2014.

[14] N. Victoir P. Friz. A note on higher dimensional p-variation. Electronic journal of Probability, $16,2011$.

[15] Nicolas Victoir Peter Friz. Multidimmensional Stochastic Processes as Rough Paths. Cambridge Studies in Advanced Mathematics, May-2009.

[16] Mark Podolskij. Ambit fields -survey and new challenges. arXiv-1405 - 1531v1, 2014.

[17] Lluis Quer-Sardanyons Samy Tindel. The 1-d stochastic wave equation driven by fractional brownian sheet. Stochastic processes and theor applications, 117:1448-1472, 2007.

[18] Nasser Towghi. Multidimmensional Extension of L. C. Young's Inequality. Journal of inequalities in pure and applied mathematics, Vol 3, issue 2, 22, Nov-2001.

[19] Henry C. Tuckwell. Numerical solutions of some hyperbolic stochastic partial differential equations with mixed derivatives including the gordon sine equation. Wave Motion, 2016.

[20] M van den Berg. Gaussian bounds for the dirichlet heat kernel. Journal of Functional Analysis, Volume 88, Issue $2,1990$.

[21] O E Barndorff-Nielsen Fred E Benth Jan Pedersen Almut E.D. Veraart. On Stochastic integration for volatility modulated Levy driven Volterra processes. arxiv 1205 2175v1, May-2012.

[22] John Walsh and R. Cairoli. Stochastic iintegral in the plane. Acta Math, 134, 1975.

[23] L.C. Young. An inequality of the holder type, connected wwith stieltjes integration. Acta Mathematica, 1936.

Fabian A. Harang, Department of Mathematics, University of Oslo, Email: fabianah@math.uio.no 